\title{
An Energy-Efficient 32-bit Multiplier Architecture in 90-nm CMOS
}

Master thesis performed in division of Electronic Devices

by

\section{Nasir Mehmood}

Thesis No: LiTH-ISY-EX--06/3852--SE

Linköping Date: 2006-09-26 
H 


\title{
An Energy-Efficient 32-bit Multiplier Architecture in 90-nm
} CMOS

\author{
Master thesis in Electronic Devices \\ at \\ Linköping Institute of Technology \\ by \\ Nasir Mehmood \\ LiTH-ISY-EX--06/3852--SE \\ Supervisor: Prof. Dr. Atila Alvandpour \\ Co-supervisor: Tekn.Lic Martin Hansson \\ Examiner: Prof. Dr. Atila Alvandpour \\ Linköping 2006-09-26
}


* 


\begin{tabular}{|c|c|c|}
\hline $\begin{array}{l}\text { Presentation date: } \\
\text { 2006-09-15 } \\
\text { Publication date: }\end{array}$ & $\begin{array}{l}\text { Division of Electronic Devices } \\
\text { Department of Electrical Engg. }\end{array}$ & Linköpings universitet \\
\hline \multirow{4}{*}{$\begin{array}{l}\text { Language } \\
\bullet \text { English } \\
\text { Swedish } \\
\text { Number of pages } \\
72\end{array}$} & \multirow{4}{*}{$\begin{array}{l}\text { Type of Publication } \\
\text { Licentiate thesis } \\
\text { - Degree thesis } \\
\text { Thesis C-level } \\
\text { Thesis D-level } \\
\text { Report } \\
\text { Other (specify below) }\end{array}$} & ISBN (Licentiate thesis) \\
\hline & & $\begin{array}{l}\text { ISRN: } \\
\text { LiTH-ISY-EX--06/3852--SE }\end{array}$ \\
\hline & & Title of series (Master thesis) \\
\hline & & Series number/ISSN () \\
\hline \multicolumn{3}{|c|}{$\begin{array}{l}\text { URL, Electronic Version } \\
\text { http://www.ep.liu.se }\end{array}$} \\
\hline \multicolumn{3}{|c|}{$\begin{array}{l}\text { Publication Title } \\
\text { Energy-efficient 32-bit multiplier architecture in 90-nm CMOS } \\
\text { Author } \\
\text { Nasir Mehmood }\end{array}$} \\
\hline \multicolumn{3}{|c|}{ 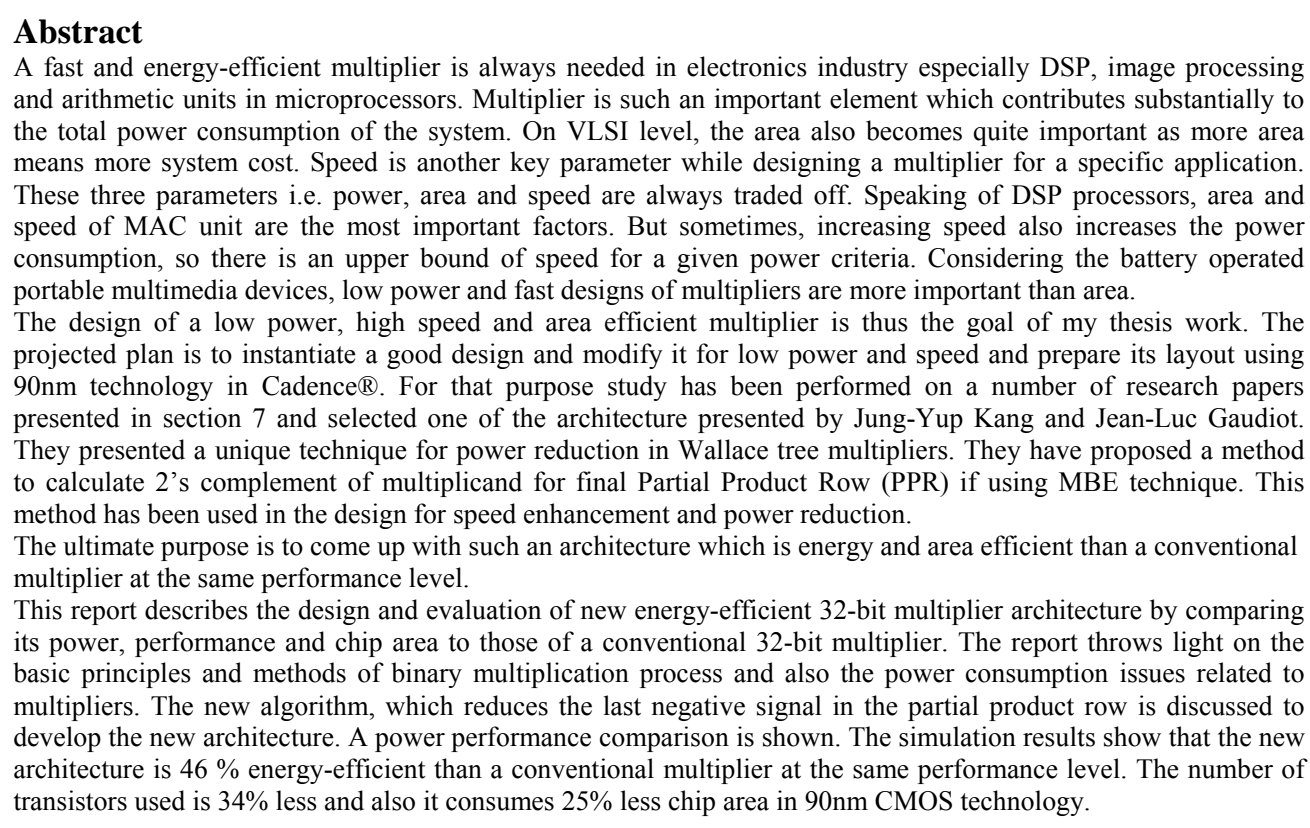 } \\
\hline \multicolumn{3}{|c|}{$\begin{array}{l}\text { Key Words: Modified Booth-encoding, carry save adder, multiplier, partial products, CMOS power, } \\
\text { two's complement, power-delay product }\end{array}$} \\
\hline
\end{tabular}


* 


\begin{tabular}{|c|} 
Team Involved \\
Prof. Dr. Atila Alvandpour \\
Supervisor \\
Division of Electronics Devices \\
Department of Electrical Engineering \\
Linköping University \\
SE-581 83 Linköping, Sweden \\
Phone : +46 (0)13-285818 \\
Mobile: +46 (0)708-485818 \\
Fax: +46 (0)13-139282 \\
Email: atila@isy.liu.se \\
Tekn. Lic. Martin Hansson \\
Ph.D. Student \\
Lic. Eng., M.Sc.EE \\
Co-Supervisor \\
Division of Electronics Devices \\
Department of Electrical Engineering \\
Linköping University \\
Office 3A:515, B building \\
Office phone: +46(0)13 282859 \\
Cell phone: +46(0)70 3444716 \\
Fax: +46(0)13 139282 \\
E-mail: martinh@isy.liu.se \\
Basir Mehmood \\
B.E Electrical (Pak) \\
Student MS SoCware \\
Office 3A:529, B building \\
Cell phone: +46(0)70 4789464 \\
Fax: +46(0)13 139282 \\
E-mail: nasme537@student.liu.se \\
mehnasir@gmail.com \\
\\
\end{tabular}


* 
Abbreviations

\begin{tabular}{|c|l|}
\hline MBE & Modified Booth Encoding \\
\hline PPR & Partial Product Row \\
\hline PPG & Partial Product Generation \\
\hline MSB & Most Significant Bit \\
\hline LSB & Least Significant Bit \\
\hline PP & Partial Product \\
\hline CSAT & Carry save adder tree \\
\hline CLA & Carry look-ahead adder \\
\hline CMOS & Complementary MOS \\
\hline & \\
\hline & \\
\hline & \\
\hline & \\
\hline & \\
\hline & \\
\hline & \\
\hline & \\
\hline & \\
\hline & \\
\hline & \\
\hline & \\
\hline & \\
\hline & \\
\hline & \\
\hline & \\
\hline & \\
\hline & \\
\hline & \\
\hline & \\
\hline & \\
\hline & \\
\hline & \\
\hline & \\
\hline & \\
\hline & \\
\hline & \\
\hline & \\
\hline & \\
\hline & \\
\hline & \\
\hline & \\
\hline & \\
\hline
\end{tabular}


H 


\begin{abstract}
A fast and energy-efficient multiplier is always needed in electronics industry especially DSP, image processing and arithmetic units in microprocessors. Multiplier is such an important element which contributes substantially to the total power consumption of the system. On VLSI level, the area also becomes quite important as more area means more system cost. Speed is another key parameter while designing a multiplier for a specific application. These three parameters i.e. power, area and speed are always traded off. Speaking of DSP processors, area and speed of MAC unit are the most important factors. But sometimes, increasing speed also increases the power consumption, so there is an upper bound of speed for a given power criteria. Considering the battery operated portable multimedia devices, low power and fast designs of multipliers are more important than area.

The design of a low power, high speed and area efficient multiplier is thus the goal of my thesis work. The projected plan is to instantiate a good design and modify it for low power and speed and prepare its layout using 90nm technology in Cadence ${ }^{\circledR}$. For that purpose study has been performed on a number of research papers presented in section 7 and selected one of the architecture presented by Jung-Yup Kang and Jean-Luc Gaudiot. They presented a unique technique for power reduction in Wallace tree multipliers. They have proposed a method to calculate 2's complement of multiplicand for final Partial Product Row (PPR) if using MBE technique. This method has been used in the design for speed enhancement and power reduction.

The ultimate purpose is to come up with such an architecture which is energy and area efficient than a conventional multiplier at the same performance level.

This report describes the design and evaluation of new energy-efficient 32-bit multiplier architecture by comparing its power, performance and chip area to those of a conventional 32-bit multiplier. The report throws light on the basic principles and methods of binary multiplication process and also the power consumption issues related to multipliers. The new algorithm which reduces the last negative signal in the partial product row is discussed to develop the new architecture. A power performance comparison is shown. The simulation results show that the new architecture is $46 \%$ energy-efficient than a conventional multiplier at the same performance level. The number of transistors used is $34 \%$ less and also it consumes 25\% less chip area in 90nm CMOS technology.
\end{abstract}


* 


\section{Preface}

This master thesis is related to the design and layout of a 32-bit low power fast multiplier. Dr. Atila Alvandpour is the project supervisor and Martin Hansson is the co-supervisor of my thesis work. Master thesis consists of 20 Swedish point's equivalent to 30 ECTS points. The duration of my thesis spans from May, 2006 to September, 2006, comprising of approximately 5 months.

The scope of thesis work includes searching for a fast Wallace tree-based multiplier, understanding of basic multiplier concepts, understanding of low power techniques, selection of an appropriate architecture, system level design, transistor level design, layout using $90 \mathrm{~nm}$ gate length transistors, simulation and performance verification, power, performance and chip area comparison with a 32-bit conventional multiplier.

Section 1 describes the basic multiplier concepts like generation of partial products, partial product reduction techniques, Modified Booth Encoding (MBE), carry save adder tree and related theory about signed/unsigned multiplier operation.

Section 2 describes the signed multiplication and various methods of representation for signed binary numbers.

Section 3 throws light on some renowned multiplier types used in today's electronic products. Array based multipliers, tree-based multiplier, bit serial and bit parallel multipliers are discussed here. Multiplier Accumulator (MAC) is also described at the end.

Section 4 deals with the power related issues in VLSI circuits generally and the multipliers in specific. It also provides knowledge about various low power techniques utilized for power reduction in VLSI circuits.

Section 5 describes the architecture of a 32-bit energy-efficient tree multiplier that has been developed for the purpose of master thesis. This section covers the previous work done by other researchers, gate level designs of all sub-modules, sign extension problem, critical path calculations and power, performance as well as chip area comparisons with a conventional multiplier.

Section 6 is about the simulation setup and results at transistor and layout levels. Section 7 covers all the references that have been consulted for the preparation of thesis report. 
*

xiv 


\section{Acknowledgments}

I would like to express my sincere gratitude to my supervisor Prof. Atila Alvandpour and co-supervisor Tekn.Lic. Martin Hansson for their esteemed guidance and assistance for my thesis work. I also thank all the staff of Electronic Devices division for being so kind and helping me during my thesis work. I am also thankful to Henrik Fredriksson and Stefan Andersson for their assistance.

I would also like to express my heartiest thanks to my father Muhammad Hussain, mother Kaneez Akhtar, my brothers Amir and Adeel, my sisters and my wife Sumaira for their affection and encouragement to complete my studies in Sweden. And specially, I feel pleasure to pay sincere gratitude to all my teachers in Govt. High School Bhakral, Fauji Foundation College, UET Taxila and Linköping University who provided me the knowledge, wisdom and inspiration due to which I am able to achieve my MS degree.

And of course I cannot forget my son Areeb whose innocent eyes are always staring at me even from thousands miles away. 
* 


\section{Table of Contents}

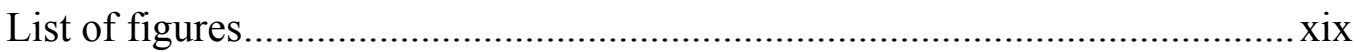

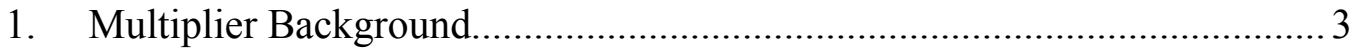

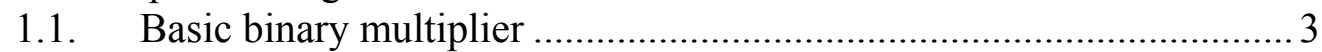

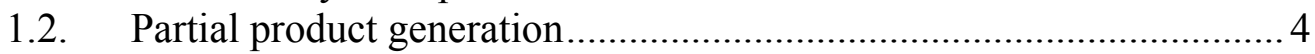

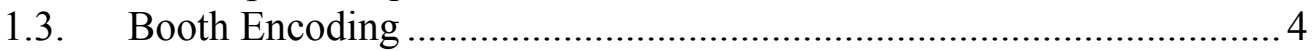

1.4. Modified Booth Encoding (MBE) ……………............................... 5

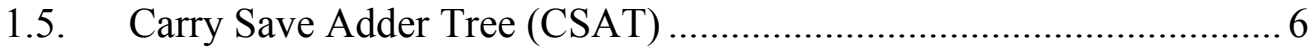

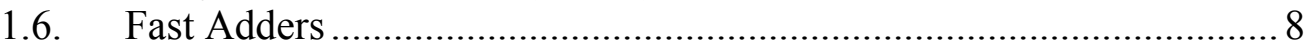

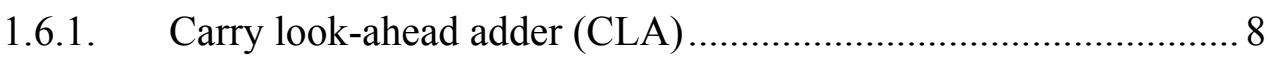

1.6.2. Simple carry skip adder ........................................................ 10

1.6.3. Multilevel carry skip adder .................................................... 12

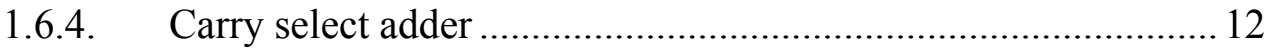

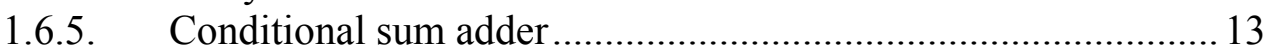

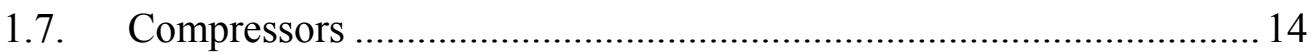

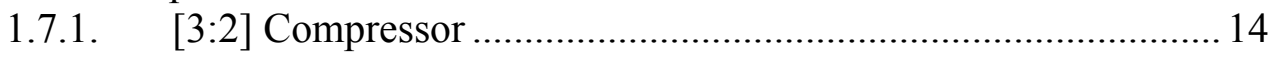

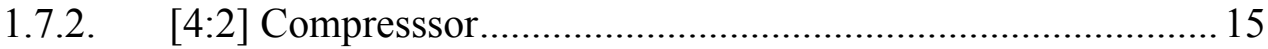

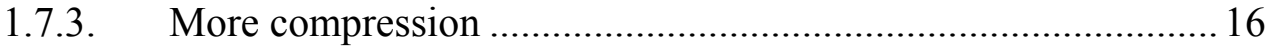

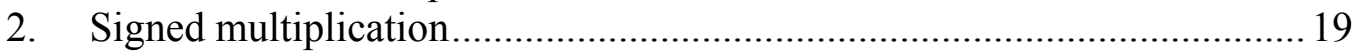

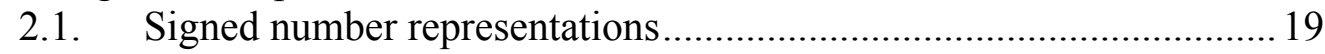

2.1.1. Sign-magnitude representation ................................................ 19

2.1.2. Complement representation ....................................................20

2.1.2.1. 1's complement representation.............................................20

2.1.2.2. 2's complement representation..............................................21

2.1.2.3. Binary offset representation................................................... 21

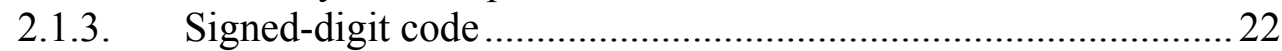

2.1.4. Canonic signed-digit code .......................................................22

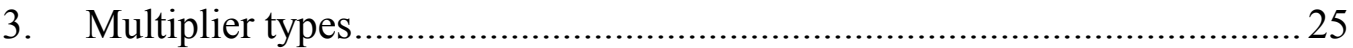

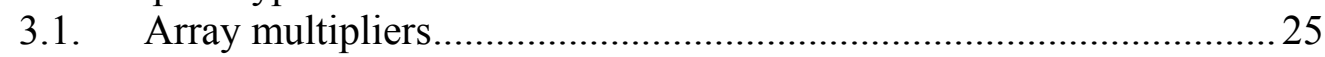

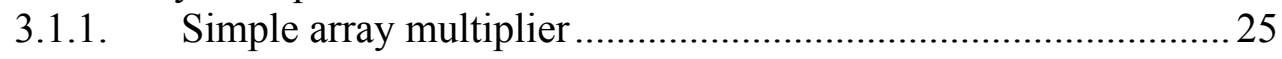

3.1.2. Double array multiplier.............................................................. 26

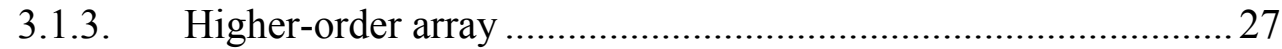

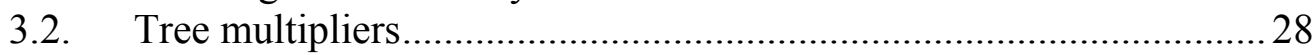

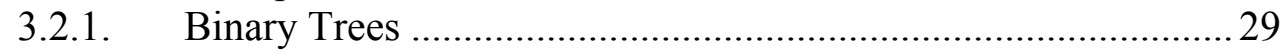

3.2.2. Balanced-Delay tree.................................................................. 30

3.2.3. Overturned-Staircase tree .......................................................... 30

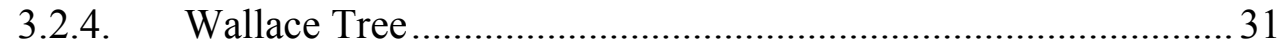

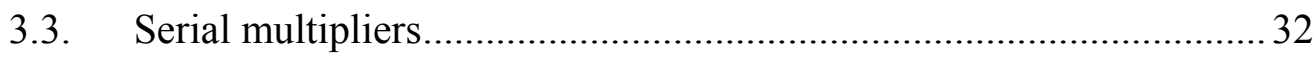

xvii 
3.3.1. Serial/parallel multiplier ......................................................... 33

3.3.2. Transposed serial/parallel multiplier ...................................... 33

3.4. Serial/Parallel Multiplier Accumulator.......................................... 34

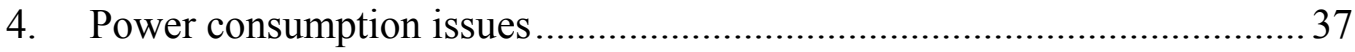

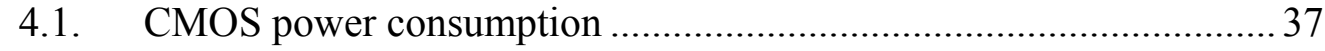

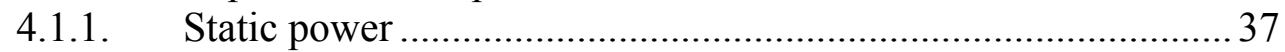

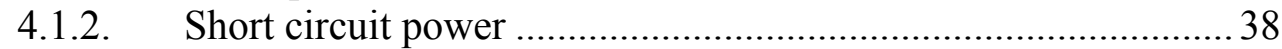

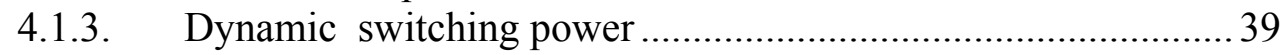

4.2. Low power techniques........................................................... 40

4.2.1. Delay balancing ............................................................... 40

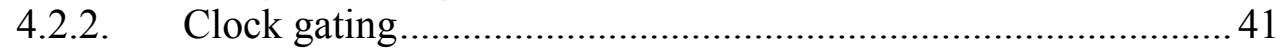

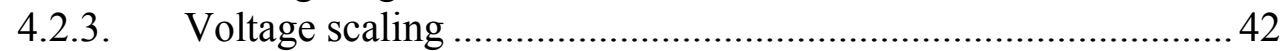

4.2.4. Transition activity reduction.................................................. 42

4.2.5. Pipelining.................................................................. 43

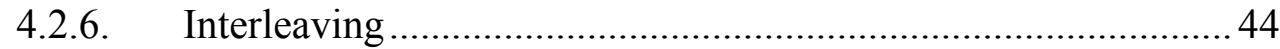

5. Energy-efficient 32 bit multiplier architecture .................................. 47

5.1. Previous related work .................................................................. 47

5.2. Architecture description............................................................. 47

5.2.1. Multiplication Algorithm...................................................... 48

5.2.2. Sign extension problem and solution..................................... 48

5.2.3. Fast method for 2's complement ........................................... 49

5.3. Development of full architecture ................................................. 51

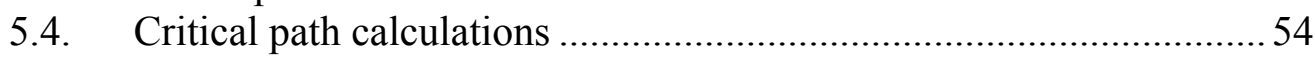

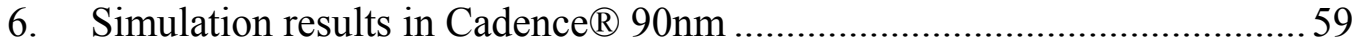

6.1. Power, performance comparison ....................................................5 59

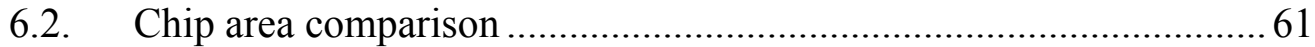

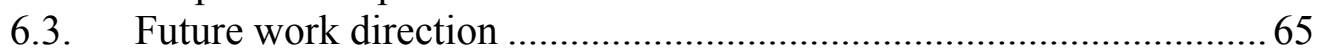

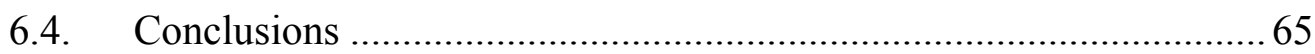

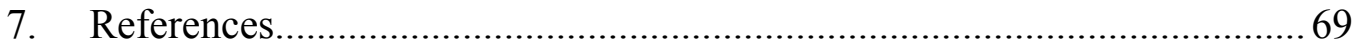




\section{List of figures}

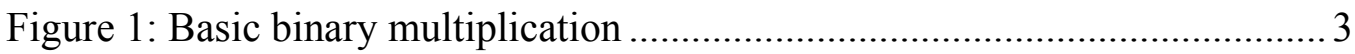

Figure 2: Signed multiplication algorithm.................................................... 3

Figure 3: Partial product generation logic ………………........................... 4

Figure 4: Multiplier bit grouping according to Booth Encoding ........................... 5

Figure 5: Bit positions in multiplier ............................................................

Figure 6: Rearranging bits in multiplier .......................................................

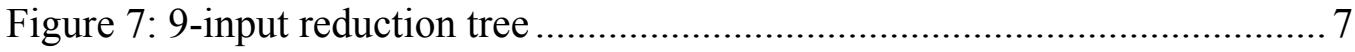

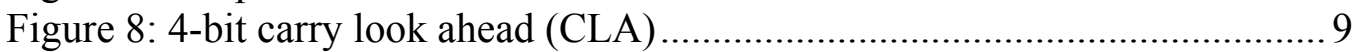

Figure 9: 4-bit ripple carry adder.............................................................. 10

Figure 10: 4-bit carry bypass adder ................................................................... 11

Figure 11: Pass transistor implementation of bypass adder.................................. 11

Figure 12: Multilevel Carry skip adder ........................................................ 12

Figure 13: Carry select module........................................................................ 13

Figure 14: 4-bit conditional sum adder........................................................... 14

Figure 15: A generic compressor...……………………............................. 14

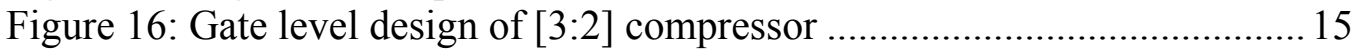

Figure 17: [4:2] compressor logic diagram [15] ............................................ 15

Figure 18: [4:2] compressor using [3:2] compressor......................................... 16

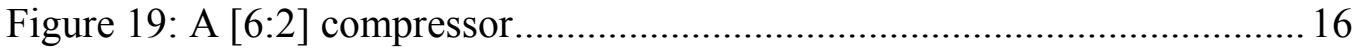

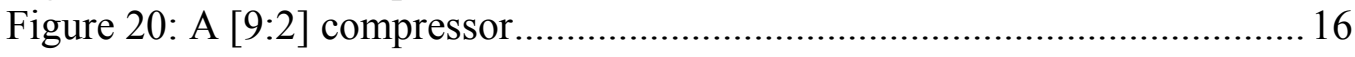

Figure 21: Array multiplier mechanism ...................................................... 25

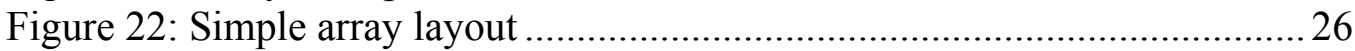

Figure 23: Partial products addition using Double array .................................... 27

Figure 24: Partial products addition using $(6,6,8,10)$ array .............................. 28

Figure 25: Partial product addition using tree topology .......................................2 29

Figure 26: Binary tree formed by 4:2 compressors …………………………..... 29

Figure 27: Overturned-staircase tree with 18 PPs ……………………….......... 31

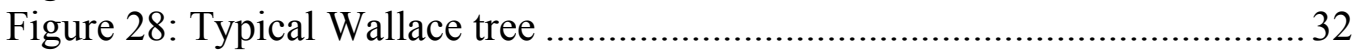

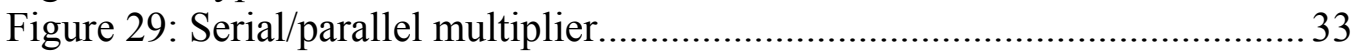

Figure 30: Transposed serial/parallel multiplier...................................................34

Figure 31: S/P multiplier accumulator................................................................. 34

Figure 32: Sources of leakage currents in CMOS [17] ....................................... 37

Figure 33: Short circuit current in CMOS circuits ............................................. 39

Figure 34: Equivalent circuit for load capacitance [18] .....................................4 40

Figure 35: Delay balancing example .................................................................. 41

Figure 36: Clock gating technique for power saving ……………………....... 42

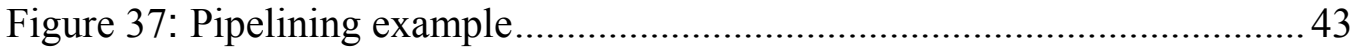

\section{xix}


Figure 38: Interleaved circuit for multiplication ............................................. 44

Figure 39: Multiplication algorithm with negative encoding ..........................48

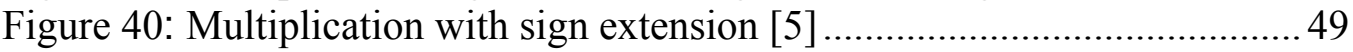

Figure 41: Two's complement conversion ................................................. 49

Figure 42: Finding conversion signals for 8 bit number ................................. 50

Figure 43: Fast logic for 2's complement computation [5] ........................... 50

Figure 44: New proposed algorithm ............................................................ 51

Figure 45: New proposed multiplier architecture........................................... 52

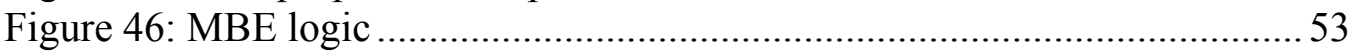

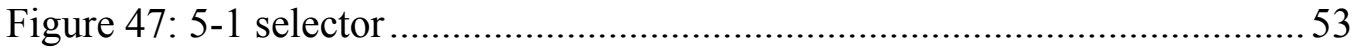

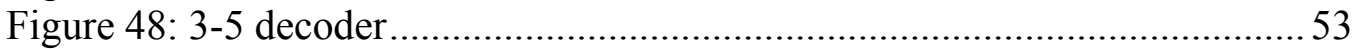

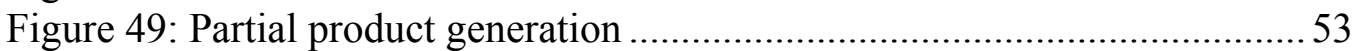

Figure 50: Critical path of conventional multiplier ...........................................5 54

Figure 51: Critical path of new architecture .................................................... 55

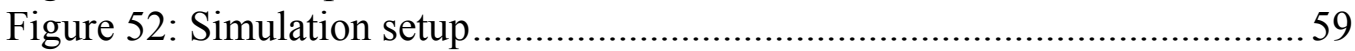

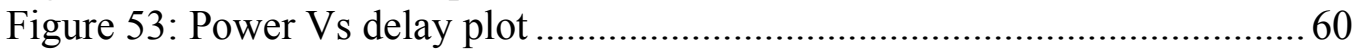

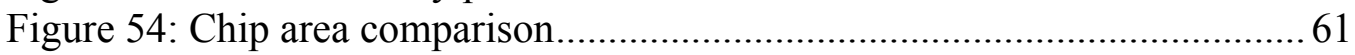

Figure 55: Simulation results @ 1.0V ...............................................................6 63

Figure 56: Simulation results @ 1.2V ................................................................. 64 


\section{PART I}

\section{BASICS OF MULTIPLICATION}


* 


\section{Multiplier Background}

\subsection{Basic binary multiplier}

The operation of multiplication is rather simple in digital electronics. It has its origin from the classical algorithm for the product of two binary numbers. This algorithm uses addition and shift left operations to calculate the product of two numbers. Two examples are presented below.

\begin{tabular}{|c|c|}
\hline $10 \times 8=80$ & $-6 \times 4=-24$ \\
\hline $\begin{array}{llll}1 & 0 & 1 & 0 \\
1 & 0 & 0 & 0\end{array}$ & $\begin{array}{llll}1 & 0 & 1 & 0 \\
0 & 1 & 0 & 0\end{array}$ \\
\hline 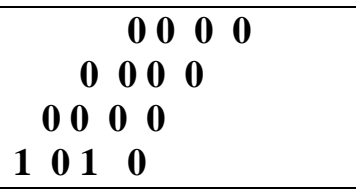 & $\begin{array}{lllllllll} & & & & & 0 & 0 & 0 & 0 \\
& & & 0 & 0 & 0 & 0 & 0 \\
1 & 1 & 1 & 0 & 1 & 0 & \\
0 & 0 & 0 & 0 & 0 & & \\
0 & 0 & 0 & & \\
\end{array}$ \\
\hline
\end{tabular}

Figure 1: Basic binary multiplication

The left example shows the multiplication procedure of two unsigned binary digits while the one on the right is for signed multiplication.. The first digit is called Multiplicand and the second Multiplier. The only difference between signed and unsigned multiplication is that we have to extend the sign bit in the case of signed one, as depicted in the given right example in PP row 3. Based upon the above procedure, we can deduce an algorithm for any kind of multiplication which is shown in Figure 2. Here, we assume that the MSB represents the sign of digit.

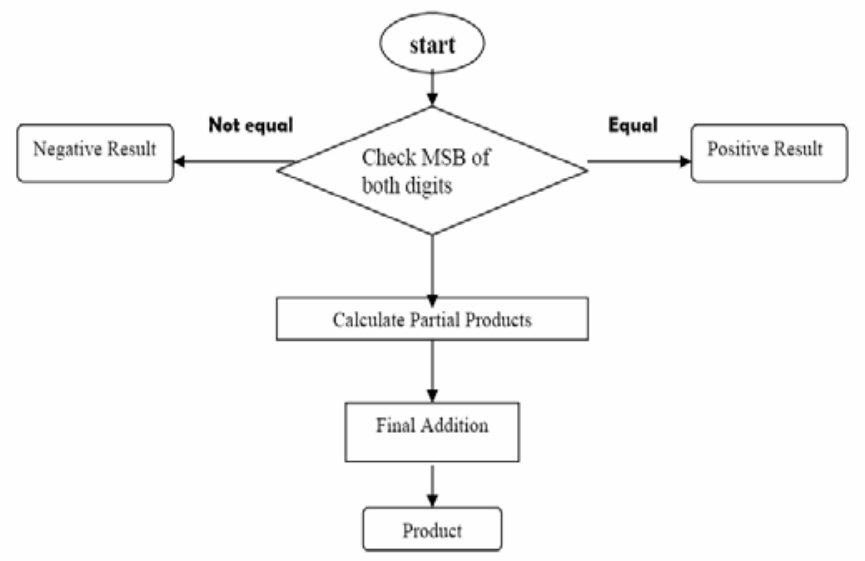

Figure 2: Signed multiplication algorithm 


\subsection{Partial product generation}

Partial product generation is the very first step in binary multiplier. These are the intermediate terms which are generated based on the value of multiplier. If the multiplier bit is ' 0 ', then partial product row is also zero, and if it is ' 1 ', then the multiplicand is copied as it is. From the 2 nd bit multiplication onwards, each partial product row is shifted one unit to the left as shown in the above mentioned example. In signed multiplication, the sign bit is also extended to the left. Partial product generators for a conventional multiplier consist of a series of logic AND gates as shown in Figure 3.

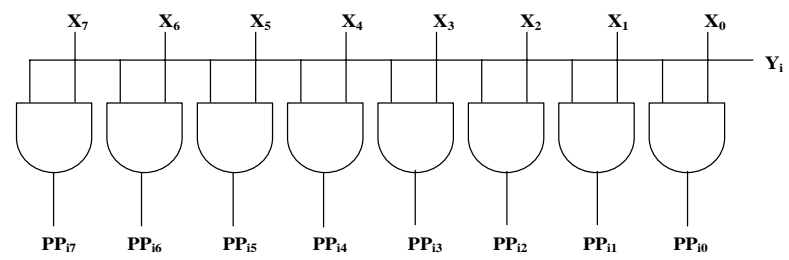

Figure 3: Partial product generation logic

Careful optimization of the partial-product generation can lead to some substantial delay and area reduction [1].

\subsection{Booth Encoding}

Booth encoding is a method used for the reduction of the number of partial products proposed by A.D. Booth in 1950. A binary number X consisting of $\mathrm{m}$ bits represented in 2's complement format can be described as [13]

$$
X=-2^{m} X_{m}+2^{m-1} X_{m-1}+2^{m-2} X_{m-2}+\ldots
$$

Rewriting Eq. 1.1 using $2^{\mathrm{a}}=2^{\mathrm{a}+1}-2^{\mathrm{a}}$ leads to

$$
X=-2^{m}\left(X_{m-1}-X_{m}\right)+2^{m-1}\left(X_{m-2}-X_{m-1}\right)+2^{m-2}\left(X_{m-3}-X_{m-2}\right) \quad \text { Eq } 1.2
$$

Considering the first 3 bits of $\mathrm{X}$, we can determine whether to add $\mathrm{Y}, 2 \mathrm{Y}$ or 0 to partial product. The grouping of $\mathrm{X}$ bits is shown in Figure 4 


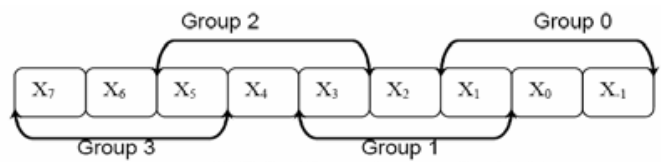

Figure 4: Multiplier bit grouping according to Booth Encoding

The multiplier $\mathrm{X}$ is segmented into groups of three bits $\left(\mathrm{X}_{\mathrm{i}+1}, \mathrm{X}_{\mathrm{i}}, \mathrm{X}_{\mathrm{i}-1}\right)$ and each group of bits is associated with its own partial product row using Table 1.

\begin{tabular}{|c|c|c|c|}
\hline \multicolumn{3}{|c|}{$X i+1 \quad X i \quad X i-1$} & Increment \\
\hline 0 & 0 & 0 & 0 \\
\hline 0 & 0 & 1 & $\mathrm{Y}$ \\
\hline 0 & 1 & 0 & $\mathrm{Y}$ \\
\hline 0 & 1 & 1 & $2 Y$ \\
\hline 1 & 0 & 0 & $-2 Y$ \\
\hline 1 & 0 & 1 & $-Y$ \\
\hline 1 & 1 & 0 & $-Y$ \\
\hline 1 & 1 & 1 & 0 \\
\hline
\end{tabular}

Table 1: Booth encoding table

\subsection{Modified Booth Encoding (MBE)}

Modified booth encoding was invented by O.L. Macsorley in 1961. MBE is an enhanced form of Booth encoding. A binary number $\mathrm{X}=\mathrm{x}_{\mathrm{m}-1}, \mathrm{x}_{\mathrm{m}-2}, \ldots \ldots, \mathrm{x}_{0}$ consisting of $\mathrm{m}$ bits represented in 2 's complement form can be mathematically expressed as

$$
X=-2^{m} x_{m-1}+\sum x_{i} 2^{i} \quad 0<i<m-2
$$

Eq 1.3

Equivalently, representation of $\mathrm{X}$ in base 4 is as follows:

$$
X=\sum d_{i} 4^{i} \quad 0<\mathrm{i}<\mathrm{m} / 2-1
$$

Eq 1.4

The digits $d_{i}$ are chosen from the ensemble $\{-2,-1,0,1,2\}$ according to Table 2 . 


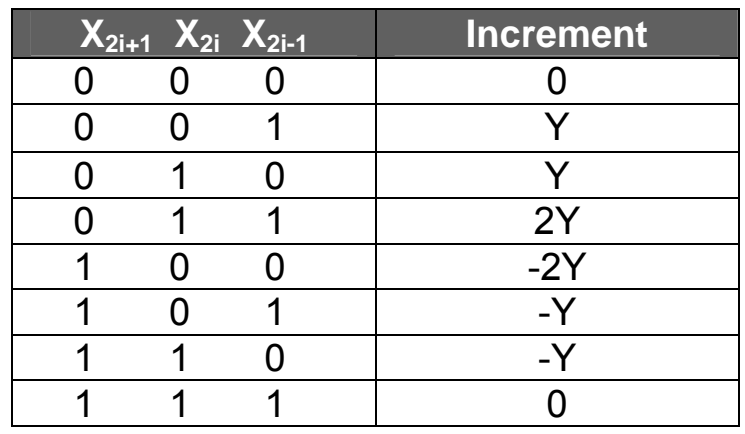

Table 2: Modified Booth encoding table

For each step $\mathrm{i}$, three bits of multiplier $\mathrm{X}$ i.e. $\mathrm{x}_{2 \mathrm{i}-1}, \mathrm{x}_{2 \mathrm{i}}, \mathrm{x}_{2 \mathrm{i}+1}$ are considered and the corresponding value of $d_{i}$ is obtained from Table 2 . There are few points to remember here.

1. Zero must always be concatenated to the right of $X$, i.e. $X_{-1}$ is considered to be 0 .

2. m must always be even.

There are two unavoidable consequences when utilizing MBE as sign extension prevention and negative encoding. The combination of these two results in the formation of one additional partial product row, which requires more hardware and the system also becomes slower [3]. This problem and its solution are discussed in section 6 .

The advantage of using MBE is that the number of partial products are reduced to $\mathrm{m} / 2$. This, in turn, reduces the hardware burden and increases the speed of multiplier.

\subsection{Carry Save Adder Tree (CSAT)}

Carry Save Adder (CSA) can be used to reduce the number of addition cycles as well as to make each cycle faster [3]. Carry save adder is also called a compressor. A full adder takes 3 inputs and produces 2 outputs i.e. sum and carry, hence it is called a 3:2 compressor. In CSA, the output carry is not passed to the neighbouring cell but is saved and passed to the cell one position down. In order to add the partial products in correct order, Carry save adder tree (CSAT) is used. An example to understand the operation of CSAT is shown in Figure 5. Suppose we have 4 partial products, each consisting of 4 bits. 
655432210 bit position

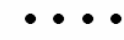

...

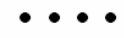

$\bullet \bullet \bullet$

Figure 5: Bit positions in multiplier

The first step is to rearrange the partial products according to bit positions as shown in Figure 6.

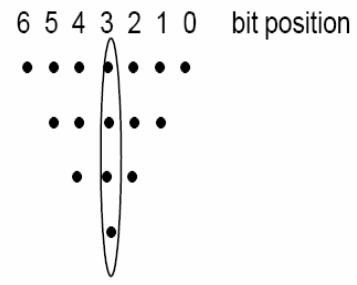

Figure 6: Rearranging bits in multiplier

The longest path consists of 4 terms at bit position 3. The final step is to determine the number of half and full adders required to complete the addition operation. A 9-input CSAT is shown in Figure 7.

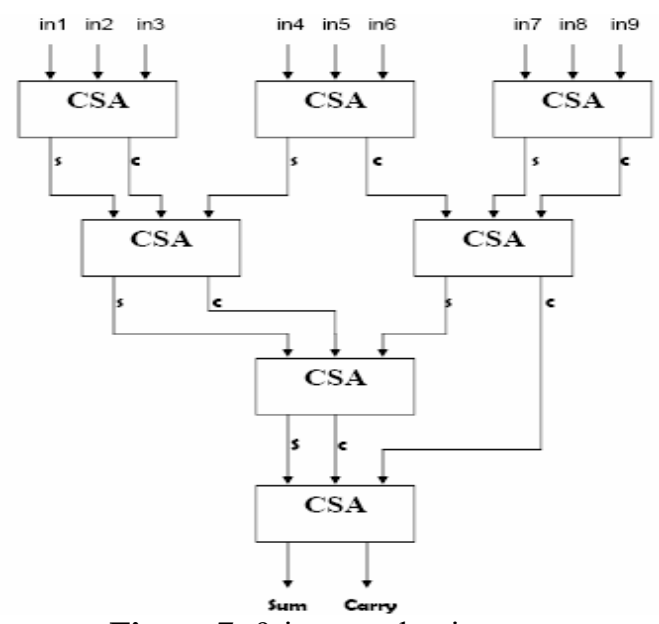

Figure 7: 9-input reduction tree

If in the level $j$ of the tree, the number of bits is $n$, then $k=n / 3$ full-adders should be used for the summation. The $\mathrm{k}$ generated carry signals are sent to the level $j+1$ of the tree $i+1$. Since the number of bits to sum has been reduced by three fold at each level, the depth of the Wallace tree is $\mathrm{O}(\log \mathrm{N})$, where $\mathrm{N}$ is the initial number of bits. 


\subsection{Fast Adders}

The final step in completing the multiplication procedure is to add the final terms in the final adder. This is normally called "Vector-merging" adder. The choice of the final adder depends on the structure of the accumulation array [1]. Following is a list of fast adders which are normally used.

1. Carry look-ahead adder

2. Simple carry skip adder

3. Multilevel carry skip adder

4. Carry- select adder

5. Conditional sum adder

6. Hybrid adder

The following sub sections discuss these adders briefly.

\subsubsection{Carry look-ahead adder (CLA)}

The concept behind the CLA is to get rid of the rippling carry present in a conventional adder design. The rippling of carry produces unnecessary delay in the circuit. For a conventional adder the expressions for sum and carry signal can be written as follows.

$$
\begin{array}{ll}
S=A \oplus B \oplus C & \text { Eq } 1.5 \\
C_{0}=A B+B C+A C & \text { Eq } 1.6
\end{array}
$$

It is useful from an implementation perspective to define $\mathrm{S}$ and $\mathrm{C}_{\mathrm{o}}$ as functions of some intermediate signals $\mathrm{G}$ (generate), D (delete) and $\mathrm{P}$ (propagate) [1]. G $=1$ means that a carry bit will be generated, $\mathrm{P}=1$ means that an incoming carry will be propagated to $\mathrm{C}_{0}$. These signals are computed as

$$
\begin{array}{ll}
G=A B & \text { Eq } 1.7 \\
P=A \oplus B & \text { Eq } 1.8
\end{array}
$$

We can write $\mathrm{S}$ and $\mathrm{C}_{0}$ in terms of $\mathrm{G}$ and $\mathrm{P}$. 


$$
\begin{aligned}
& C_{0}(G, P)=G+P C \\
& S(G, P)=P \oplus C
\end{aligned}
$$

For an N-bit adder, the following relation holds for the carry signal.

$$
C_{0, k}=G_{k}+P_{k} C_{0, k-1}
$$

In a fully expanded form [1], we have

$$
C_{0, k}=G_{k}+P_{k}\left(G_{k-1}+P_{k-1}\left(\ldots . .+P_{1}\left(G_{0}+P_{0} C_{i, 0}\right)\right)\right) \quad \text { Eq } 1.12
$$

For $\mathrm{k}=4$, the logic expressions are as

$$
\begin{aligned}
& C_{4}=G_{3}+G_{2} P_{3}+G_{1} P_{2} P_{3}+G_{0} P_{1} P_{2} P_{3}+C_{0} P_{0} P_{1} P_{2} P_{3}=G_{3}+P_{3} C_{3} \\
& C_{3}=G_{2}+G_{1} P_{2}+G_{0} P_{1} P_{2}+C_{0} P_{0} P_{1} P_{2}=G_{2}+P_{2} C_{2} \\
& C_{2}=G_{1}+G_{0} P_{1}+C_{0} P_{0} P_{1}=G_{1}+P_{1} C_{1} \\
& C_{1}=G_{0}+C_{0} P_{0}
\end{aligned}
$$

This expanded relationship is used to implement N-bit adder. The carry and sum outputs are independent of the previous bits. The ripple effect has been eliminated [1]. The logic design of a 4-bit CLA is shown in Figure 8.

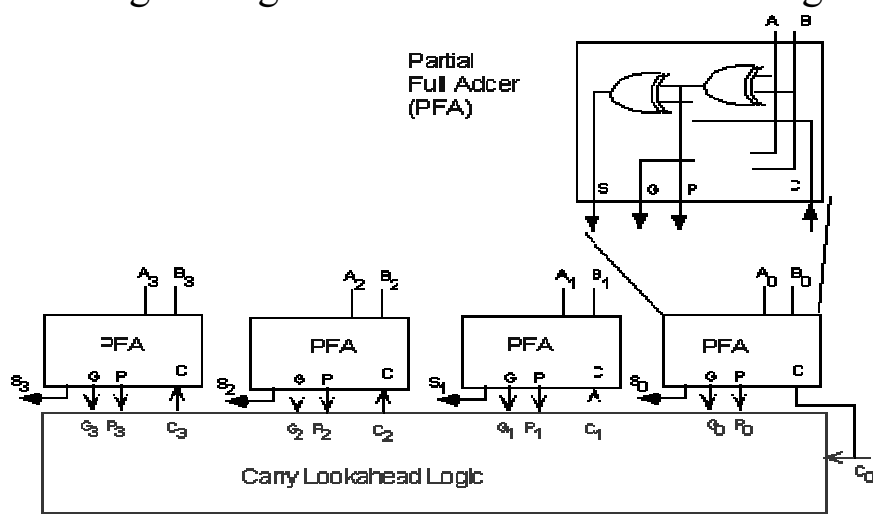

Figure 8: 4-bit carry look ahead (CLA) 
Full carry CLA is impractical for wide words. Since wide gates and large stacks display poor performance, the CLA computation has to be limited to up to 2 or 4 bits in practice. For example, the equation for $\mathrm{C} 31$ consists of 32 product terms, the largest of which contains 32 literals. Thus the required AND and OR functions must be realized by tree networks, leading to increased latency and cost [3]. Two schemes for managing this complexity are 1) High-radix addition 2) Multilevel lookahead. Multilevel lookahead is the most widely used technique for large CLAs.

\subsubsection{Simple carry skip adder}

The simple carry skip adder uses logic to bypass the rippling carry, depending on the status of Propagate $(\mathrm{P})$ signals. Consider a simple 4-bit ripple carry adder as shown in Figure 9.

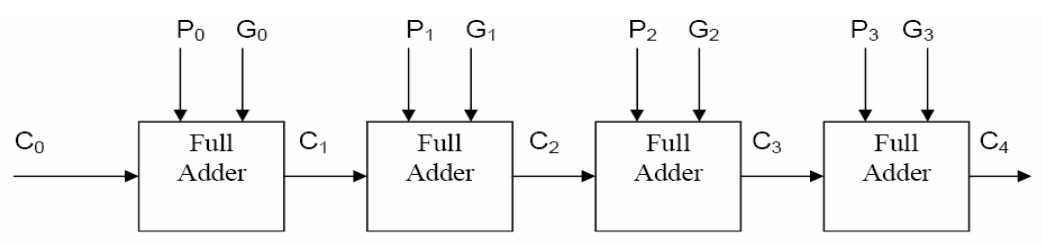

Figure 9: 4-bit ripple carry adder

The propagate signals are "high" if $\mathrm{A}$ and $\mathrm{B}$ are ' 0 ' and ' 1 ' and vice versa. If all $\mathrm{P}$ signals are ' 1 ' and $\mathrm{C}_{0}=1$, then all intermediate carry signals will be ' 1 '. In other words, $\mathrm{C}_{4}$ is equal to $\mathrm{C}_{0}$. We can write that [1]

$$
\text { If } \mathrm{P}_{0} \mathrm{P}_{1} \mathrm{P}_{2} \mathrm{P}_{3}=1 \text { then } \mathrm{C}_{4}=\mathrm{C}_{0}
$$

This operation can be used to construct a carry-bypass adder. A simple 4-bit structure is shown in Figure 10. 


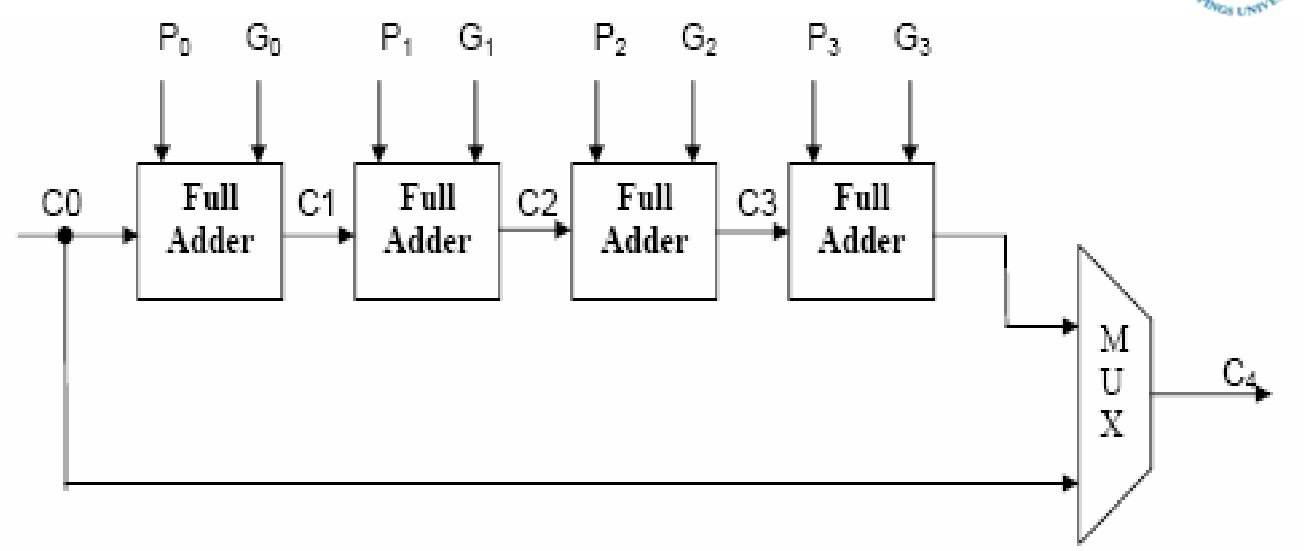

Figure 10: 4-bit carry bypass adder

Figure 11 shows the transistor level implementation of 4-bit carry-bypass adder using pass transistor logic.

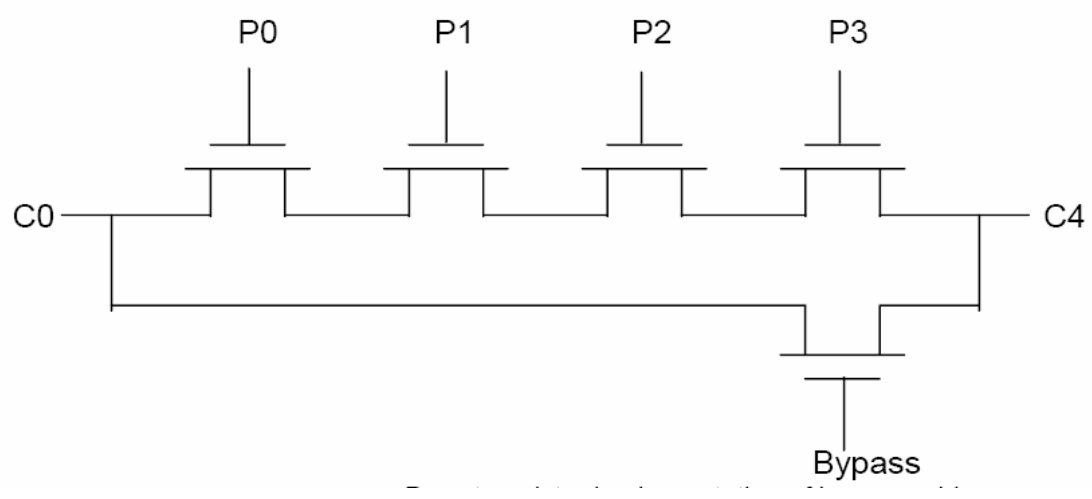

Figure 11: Pass transistor implementation of bypass adder

To compute the delay of an N-bit adder, we assume that there are N/M equal length bypass stages each containing $\mathrm{M}$ bits. An approximate expression can be derived below.

$$
T_{p}=t_{\text {setup }}+[N / M-1] t_{\text {bypass }}+[M-1] t_{\text {carry }}+t_{\text {sum }} \quad \text { Eq } 1.17
$$

Where

$t_{\text {setup }}$ : $\quad$ time to create the generate and propagate signals

$t_{\text {carry }}$ : $\quad$ propagation delay through a single bit 
$t_{\text {bypass }}$ : $\quad$ propagation delay through the bypass multiplexer

$t_{\text {sum }}$ : $\quad$ time to generate the final sum

\subsubsection{Multilevel carry skip adder}

The idea of a simple carry skip adder can be extended for N-bit adder. We assume that the total adder is divided in (N/M) equal-length bypass stages, each of which contains $\mathrm{M}$ bits. The bits are divided in blocks of 4-bits. The diagram of 12-bit adder is shown in Figure 12.

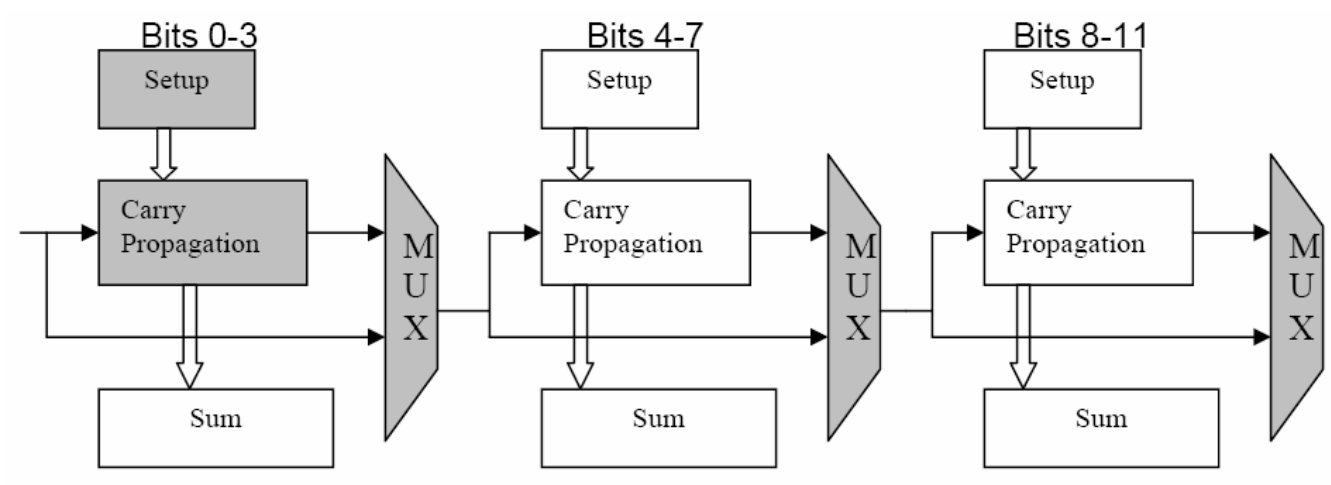

Figure 12: Multilevel Carry skip adder

\subsubsection{Carry select adder}

Carry select adder is based on anticipation of output carry for two possible values of input carry. Once the real value of the incoming carry is known, the correct result is easily selected with a simple multiplexer stage [1]. Carry select adder can be implemented in two different ways 1) Linear carry select adder 2) Square-root carry select adder. Consider the block of adders, which is adding bits $\mathrm{k}$ to $\mathrm{k}+3$. Instead of waiting on the arrival of the output carry of bit $\mathrm{k}-1$, both the 0 and 1 possibility is analysed. A multiplexer can be used to select either of the results when Co,k-1 settles. The hardware overhead of the carry select adder is an additional carry path and a multiplexer. A basic structure of linear carry select adder is shown in Figure 13. 


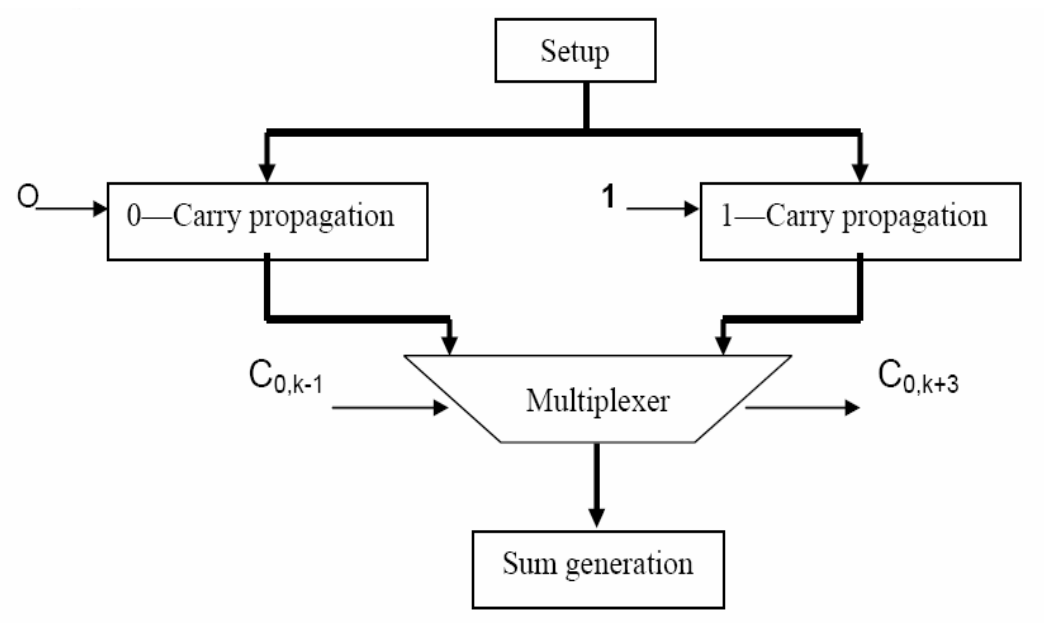

Figure 13: Carry select module

A full carry select adder is now built using a chain of equal-length adder stages, as in carry bypass adder. The propagation delay of N-bit adder with M-bit stages can be determined as follows.

$$
T_{\text {add }}=t_{\text {setup }}+M t_{\text {carry }}+[N / M] t_{\text {mux }}+t_{\text {sum }} \quad \text { Eq } 1.18
$$

The carry select adder can be optimized to reduce the delay of critical path by making the adder stages progressively longer than the previous ones. This architecture results in square root dependence and is called square root carry select adder. For example, the first stage can add 2 bits, the second consists of 3 , and the third has 4 and so on.

\subsubsection{Conditional sum adder}

In conditional sum adder, two sets of outputs are generated in parallel for a given group of input bits. Each set consist of $\mathrm{N}$ bits and a carry output. One set calculates the result by assuming that the input carry is zero while the other assumes that the input carry is one. Once the correct value of carry is known, then we just need to select the correct set of outputs out of two without waiting for the carry signal to ripple through $\mathrm{N}$ bit positions. Figure 14 shows a 4-bit conditional sum adder [14]. 


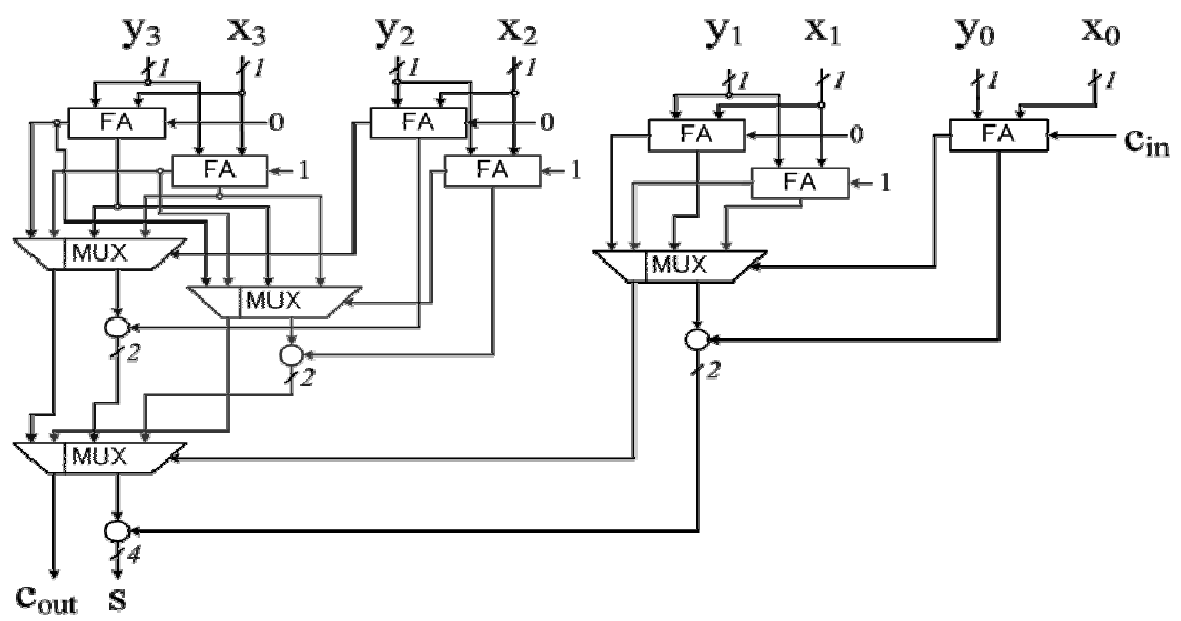

Figure 14: 4-bit conditional sum adder

\subsection{Compressors}

Compressors are mostly used in multipliers to reduce the operands while adding terms of partial products. A compressor $\mathrm{C}_{\mathrm{i}}$ is a combinatorial device that compresses $\mathrm{N}$ input lines in the position i to 2 output lines i.e. sum and carry. In addition, there are $\mathrm{L}$ inputs lines coming to the compressor to different levels $\mathrm{j}$. Figure 15 shows a simple compressor.

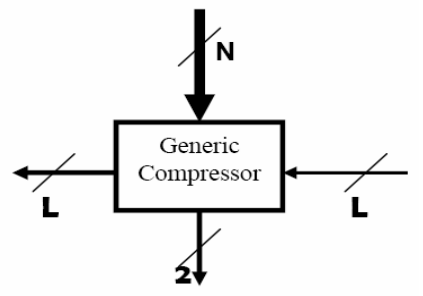

Figure 15: A generic compressor

\subsection{1. [3:2] Compressor}

A [3:2] compressor is basically a Full adder. It has 3 inputs $i_{1}, i_{2}$ and $i_{3}$ to be summed up and provides 2 outputs (sum and carry). Gate level diagram of [3:2] compressor is shown in Figure 16. 


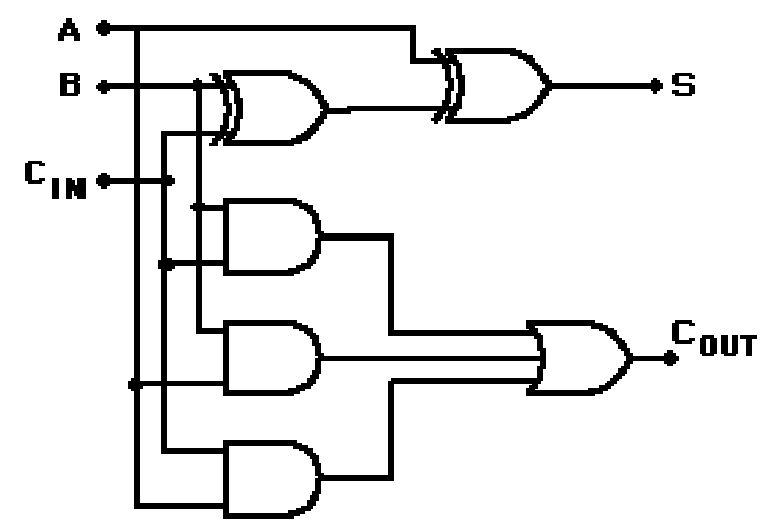

Figure 16: Gate level design of [3:2] compressor

\subsection{2. [4:2] Compresssor}

A [4:2] compressor has 4 input lines $i_{1}, i_{2}, i_{3}$ and $i_{4}$ that must be summed and has two output lines $\mathrm{s}$ and $\mathrm{c}$, which are so called results of compression. The additional lines are input and output carries. The gate level design of a [4:2] compressor is shown in Figure 17.

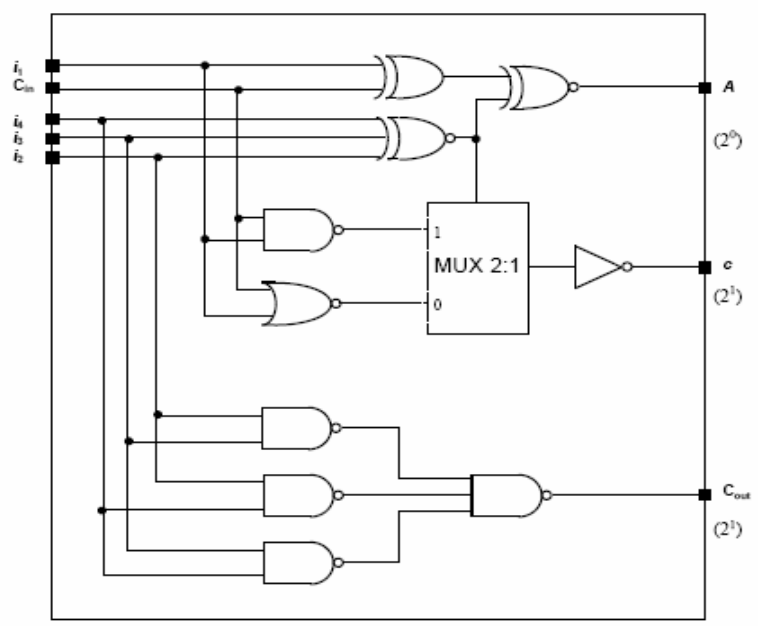

Figure 17: [4:2] compressor logic diagram [15]

A [4:2] compressor can also be designed using two [3:2] compressors as shown in Figure 18. 


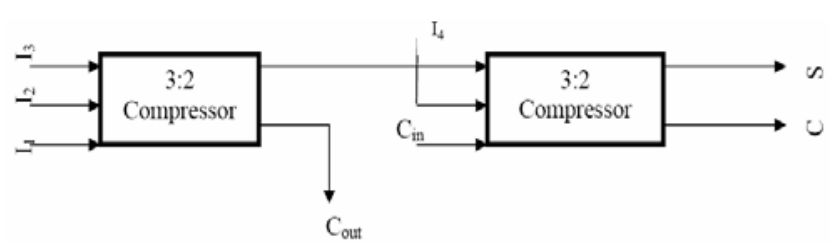

Figure 18: [4:2] compressor using [3:2] compressor

\subsubsection{More compression}

Based on the previous discussion, further complex compressors can be built by using basic compressors like [3:2] and [4:2] compressors. For example, a [6:2] compressor can be built using two [3:2] and one [4:2] compressors.

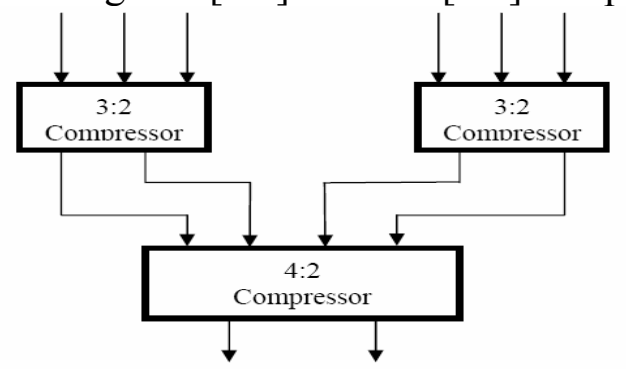

Figure 19: A [6:2] compressor

Similarly, a [9:2] compressor can be designed using three [3:2] and one [6:2] compressors and is shown in

Figure 20.

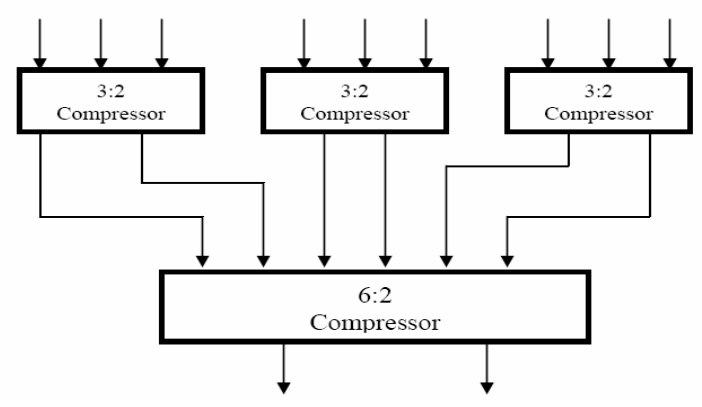

Figure 20: A [9:2] compressor 


\section{PART II}

\section{SIGNED MULTIPLICATION}


* 


\section{Signed multiplication}

There are two kinds of multipliers i.e. unsigned and signed. In unsigned multiplier, it is assumed that both the multiplier and the multiplicand are positive integers, while in signed multipliers, the operands bear signs which is represented by the MSB of each operand. A signed multiplier is a little bit more complex than an unsigned one due to the possibility that any number can be negative changing the result. The sign bit creates some troubles while compressing the partial products. The sign bit of a partial product row has to be extended all the way to the MSB position which would require the sign bit to drive that many output loads. This makes the partial product rows unequal in length [3].

\subsection{Signed number representations}

Performance of the multiplier with respect to speed, chip area and power dissipation also depends upon the number representation used [4]. For fixedpoint numbers one of the following representations can be used.

- Sign-magnitude representation

- Complement representation

o 1's complement representation

o 2's complement representation

o Binary offset representation

- Signed-digit code

- Canonic signed digit code

These representations will be discussed in the subsequent sections.

\subsubsection{Sign-magnitude representation}

In sign-magnitude representation, a number is expressed so that the MSB represents the sign and the remaining bits represent the magnitude of that number. Mathematically it can be represented as

$$
X=\left(1-2 x_{0}\right) \sum_{i=1}^{W_{d}-1} x_{i} .2^{-i}
$$


Where $\mathrm{W}_{\mathrm{d}}$ is the binary number width in bits.

A normalized value is in the range $-1 \leq x \leq 1$. Hence, the binary point is placed immediately to the right of $\mathrm{x}_{0}$. In this representation, the numbers having the same magnitudes are represented by the same binary word except the sign bit [4]. For example

$$
\begin{aligned}
& (+0.828125)_{10}=(0.110101)_{2} \\
& (-0.828125)_{10}=(1.110101)_{2}
\end{aligned}
$$

An important phenomenon is that " 0 " can be represented in two different representation i.e. +0 or -0 . This may complicate the design of ALU since it has to check for two different zeros. One drawback is that values +1 and -1 don't have any representation in this system. A major disadvantage is that the addition and subtraction depend on the signs of the operands.

\subsubsection{Complement representation}

Addition and subtraction can be performed independent of the sign of the operands by using the complement representations. There are three types of complement representation i.e. 1's complement, 2's complement and binary offset representation.

\subsubsection{1's complement representation}

In 1's complement format, a signed number is represented by a sign bit at MSB position; the remaining bits represent the magnitude. The magnitude bits are in 1 's complement format for negative numbers and true for positive numbers. Some examples are given below

$$
\begin{aligned}
& +(12)_{10}=01100_{1 \mathrm{c}} \\
& -(12)_{10}=10011_{1 \mathrm{c}} \\
& \quad(0)_{10}=(0.00000)_{1 \mathrm{c}} \text { or }(1.11111)_{1 \mathrm{c}}
\end{aligned}
$$

The values +1 and -1 still can not be represented by this format. Numbers having the same magnitudes but different signs are represented by different binary words as shown above. For $\mathrm{x}>0$, 1 's complement has the same representation as sign-magnitude representation. For $\mathrm{x}<0$, the values are the bit complements of the positive values. A change of sign is more easier in sign- 
magnitude form compared to binary offset and two's complement representations, since using these representations, changing the sign of a number is done by taking the bit complement [4]. Multiplication is more complicated in 1 's complement representation than 2's complement or binary offset.

\subsubsection{2's complement representation}

Two's complement is the most widely used representation in digital arithmetic. In this representation, a negative number is represented by a sign bit at MSB position and magnitude bits in 2's complement form. The value of a normalized binary word is represented as

$$
X=-x_{0}+\sum_{i=1}^{W_{d}-1} x_{i} \cdot 2^{-i}
$$

For $\mathrm{x}>0$, two's complement has the same binary word as sign-magnitude representation. The negative number is represented by taking the two's complement of the magnitude bits. Some examples are shown below.

$$
\begin{gathered}
+(12)_{10}=01100_{2 \mathrm{c}} \\
-(12)_{10}=10100_{2 \mathrm{c}} \\
(0)_{10}=(0.00000)_{2 \mathrm{c}}
\end{gathered}
$$

The advantage of 2's complement is that zero has only one representation which makes the design of arithmetic units easier.

\subsubsection{Binary offset representation}

This representation is similar to 2's complement with the only difference that the sign bit is complemented. For example

$$
\begin{gathered}
+(12)_{10}=11100_{\mathrm{BO}} \\
-(12)_{10}=00100_{\mathrm{BO}} \\
\quad \text { (0) } 10=1.00000_{\mathrm{BO}}
\end{gathered}
$$




\subsubsection{Signed-digit code}

In signed-digit code, a number $\mathrm{x}$, is represented by

$$
X=\sum_{i=0}^{W_{d}-1} x_{i} \cdot 2^{-i} \quad \text { where } x_{i}=-1,0 \text { or }+1
$$

For example, the decimal number $(15 / 32)_{10}=(0.01111)_{2 \mathrm{c}}$ can be represented by $(0.1000-1)_{\mathrm{SDC}}$. The decimal number $(-15 / 32)=(1.10001)_{2 \mathrm{c}}$ can be represented by $(0 .-10001)$ SDC or $(0.0-1-1-1-1)$ SDC.

The signed-digit code is not unique. This causes problems when a number is to be quantized. An SDC number is therefore converted to a conventional representation before it is quantized. In practice, it may be quite costly to convert SDC numbers for the necessary quantization and overflow checks in every recursive loop [4].

\subsubsection{Canonic signed-digit code}

A canonic signed-digit code (CSDC) is a special form of SDC in which each number has a unique number representation. $A$ number $\mathrm{x}$ in the range $\{-4 / 3+Q \leq x \leq 4 / 3+Q\}$, where $\mathrm{Q}=2-\mathrm{W}, \mathrm{W}=\mathrm{W}_{\mathrm{d}}-1$ for $\mathrm{W}_{\mathrm{d}}=$ odd and $\mathrm{W}=$ $\mathrm{W}_{\mathrm{d}}-2$ for $\mathrm{W}_{\mathrm{d}}=$ even, is represented in CSDC by

$$
X=\sum_{i=0}^{W_{d}-1} x_{i} \cdot 2^{-i} \text { where } x_{i}=-1,0 \text { or }+1 \quad \text { Eq } 2.4
$$

Where no two consecutive digits are nonzero i.e.

$$
\mathrm{x}_{\mathrm{i}} \cdot \mathrm{x}_{\mathrm{i}+1}=0,0 \leq i \leq W_{d}-2
$$

For example, the number (15/32)10 is represented by $(0.1000-1)$ CSDC and ($15 / 32) 10$ by $(0 .-10001)$ CSDC [4]. 


\section{PART III}

\section{TYPES OF MULTIPLIERS}


* 


\section{Multiplier types}

Multipliers are categorized relative to their applications, architecture and the way the partial products are produced and summed up. Based on all these, a designer might find following types of multipliers.

\subsection{Array multipliers}

In array multipliers, the counters and compressors are connected in a serial fashion for all bit slices of the Partial Product parallelogram. As can be seen in Figure 21, the array topology is a two-dimensional structure that fits nicely on the VLSI planar process [2].

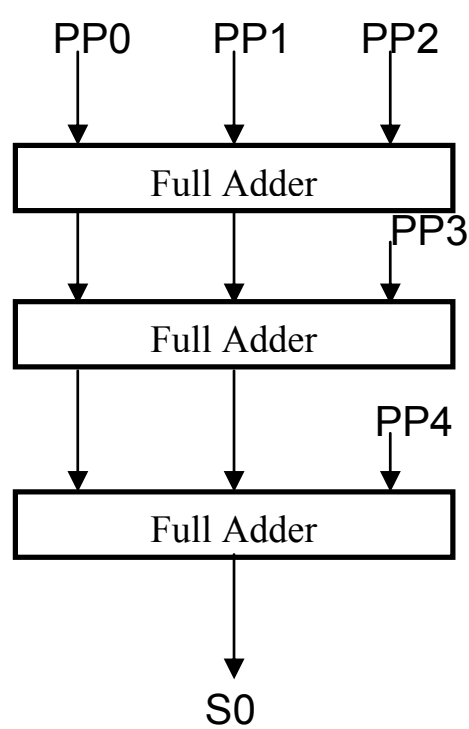

Figure 21: Array multiplier mechanism

There are several possible array topologies including simple, double and higherorder arrays.

\subsubsection{Simple array multiplier}

In this type of array, the output of each row of counters ( $3: 2$ compressors) is the input to the next row of counters [2]. In the simple array, each row of [3:2] compressors adds a partial product to the partial sum, generating a new partial 
sum and a sequence of carries. The delay of the array depends on the depth of the array. Therefore, the summing time for the simple array is N-2 [3:2] compressor delays, where $\mathrm{N}$ is the number of partial products. The drawback of this type of array is the hardware is underutilized. The counters are used only once in the calculation of the result, for the remaining time, they are idle. This drawback can be diminished by pipelining the array so that several multiplications can occur simultaneously. Pipelining would increase the throughput of the multiplier, but would also increase the latency and area of the multiplier. A fully pipelined array is normally avoided, since the array would be faster than the clock of processor. Figure 22 depicts the layout of a simple array topology. The dots represent the partial products.

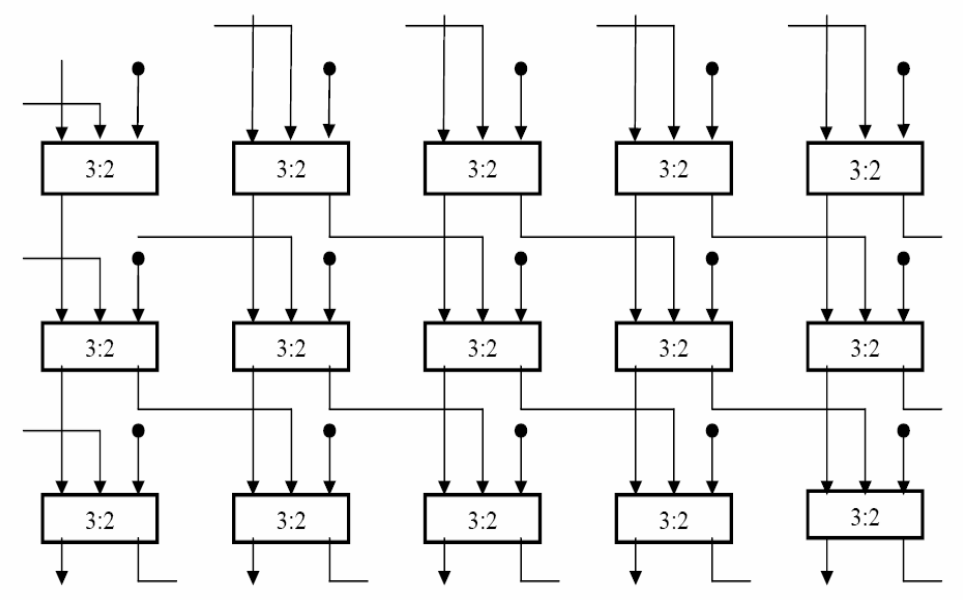

Figure 22: Simple array layout

\subsubsection{Double array multiplier}

The double array design is faster than a simple array one. In this type of array, the delay required to produce the result for the simple array can be halved by adding partial products in two parallel rows. The odd-numbered PPs are added in one row while the other row adds the even-numbered PPs [2]. When all the Partial products are accumulated, the two partial sums are combined using a [4:2] compressor. The double array also consists of rows of [3:2] compressors. However, the output of the counter is the input to the row after the next one. The delay required to reduce the partial products is

$$
[\mathrm{N} / 2 \text {-2] [3:2] compressors }+1[4: 2] \text { compressor }
$$


Figure 23 clearly explains the basic idea of adding PPs using double array.

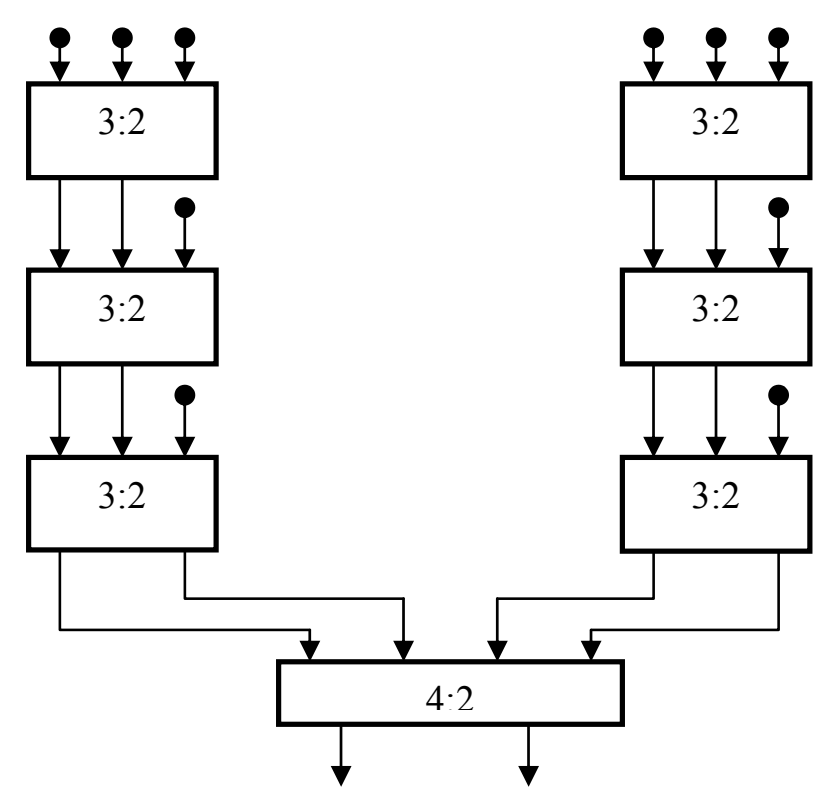

Figure 23: Partial products addition using Double array

\subsubsection{Higher-order array}

In higher-order arrays, more additions are performed concurrently thereby reducing the delay to produce the final result. The idea is to partition the array into more sub-arrays and use [4:2] compressors to combine the sub-arrays. This is accomplished by connecting progressively longer simple array together. The [4:2] compressor is used between a simple array and the other arrays when the delay of the simple array is equal to the total delay of the combined arrays [2].

The higher-order arrays are classified according to the number of partial products in each sub-array. For example, the $[6,6,8,10]$ array combines two simple arrays that each reduce six PPs using a [4:2] compressor. The resulting structure is combined with a simple array that reduces 8 PPs. Finally, the resulting structure is combined with a simple array that sums 10 PPs [2]. Consider the $[6,6,8]$ high-order array. The delay for each of the simple arrays that reduce 6 PPs is four [3:2] compressor delays plus one [4:2] compressor delay. So the resulting delay is

$$
4_{(3: 2)} \operatorname{comp}+1_{(4: 2)} \text { comp }=6_{(3: 2)} \text { comp }
$$


This is connected to the simple 8PP array which has a delay of six [3:2] compressors. Hence, the delay of the larger sub array is approximately equal to that of the combined one. Figure 24 shows a complex array.

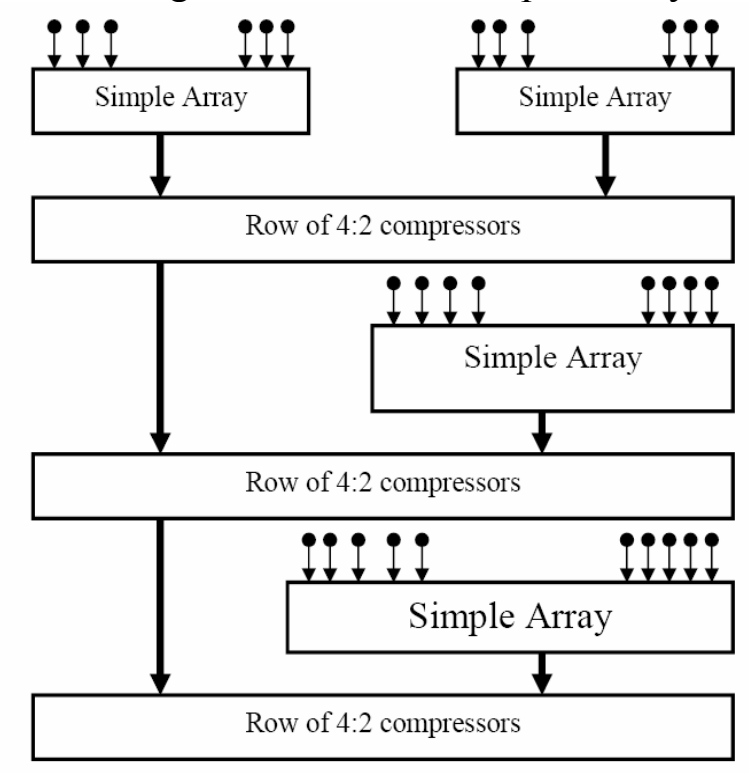

Figure 24: Partial products addition using $(6,6,8,10)$ array

\subsection{Tree multipliers}

In order to speed up the process of addition of partial products, tree based structure is used. In tree architecture, the compressors are connected for each bit slice in the PP parallelogram. Normally, they are used in parallel. Although the trees are faster than arrays, they both use the same number of compressors to reduce the partial products. An example is shown in Figure 25. 


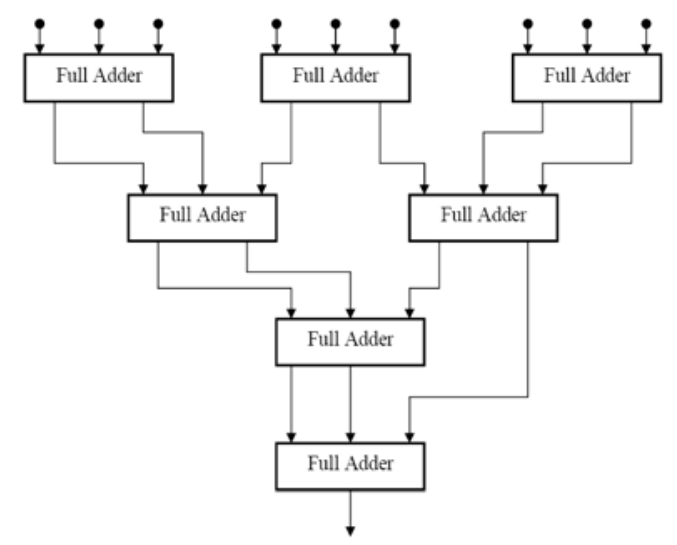

Figure 25: Partial product addition using tree topology

The first tree structure was introduced by Wallace. Wallace showed that PPs can be reduced by connecting [3:2] compressors in parallel in a tree topology. The regular trees include binary, balanced-delay and overturned-staircase trees as well as [9:2] compressors.

\subsubsection{Binary Trees}

The [4:2] compressor designed by Shen and Weinberger in 1978, is the basis for binary tree multipliers. A [4:2] compressor can be designed by connecting two [3:2] compressors in series. Although it can also be built in a smart and fast way by using 3 XORs in the critical path. This reduces the [4:2] compressor delay by approximately $25 \%$. Figure 26 shows a 2-dimensional view of a bit slice of the 16-bit binary tree. The [4:2] compressor reduces the PPs in logarithmic time, because of its 2-to-1 reduction ratio [2].

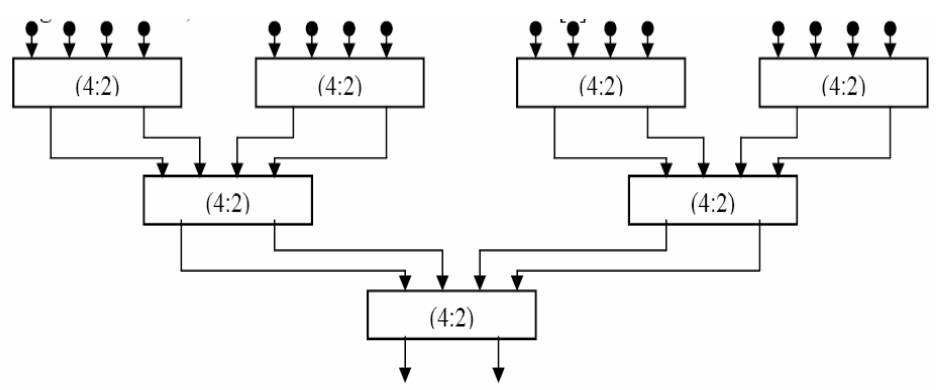

Figure 26: Binary tree formed by $4: 2$ compressors 


\subsubsection{Balanced-Delay tree}

This architecture was proposed by Zuras and McAllister and is abbreviated as ZM tree. It is a regular topology, in which any tree is constructed from a few building blocks. ZM tree is constructed by connecting progressively longer serial chains of [3:2] counters in a tree. When the delay of the longest chain is equal to the delay of the remainder of the tree, a new chain is started [2]. This tree is a balanced-delay tree of type- $1\left(\mathrm{ZM}_{1}\right)$. Higher-order balanced trees are built by recursively replacing the serial chains of [3:2] compressors with a balanced-delay tree of $\mathrm{ZM}_{1}$. The balanced-delay tree of type 1 reduces the partial products in $\mathrm{O}(2 \sqrt{\mathrm{N}})$ compressor stages.

\subsubsection{Overturned-Staircase tree}

The Overturned-Staircase (OS) tree is a regular structure and is designed recursively. It achieves the same number of compressor levels as Wallace for many values of the PPs. This tree is constructed from a root and the body. The root is the last [3:2] compressor. The body is a recursive concatenation of compressors. A body of height $\mathrm{k}$, where $\mathrm{k}$ is the maximum number of [3:2] counters in series, is constructed from a body of height k-1 and a branch of serially connected [3:2] compressors. The smaller body and the branch are connected using a connector. The connector is made of two [3:2] compressors, each of which takes five inputs and produces three outputs [2]. By this way, OS tree of type 1 is constructed. An $\mathrm{OS}_{2}$ tree is built by replacing each branch in the tree with an $\mathrm{OS}_{1}$ tree that has the same delay. This method can be further extended to build even higher-order OS trees by replacing the branches in the $\mathrm{OS}_{2}$ tree with $\mathrm{OS}_{1}$ trees. An example of OS tree reducing 18 PPs is shown in Figure 27. 


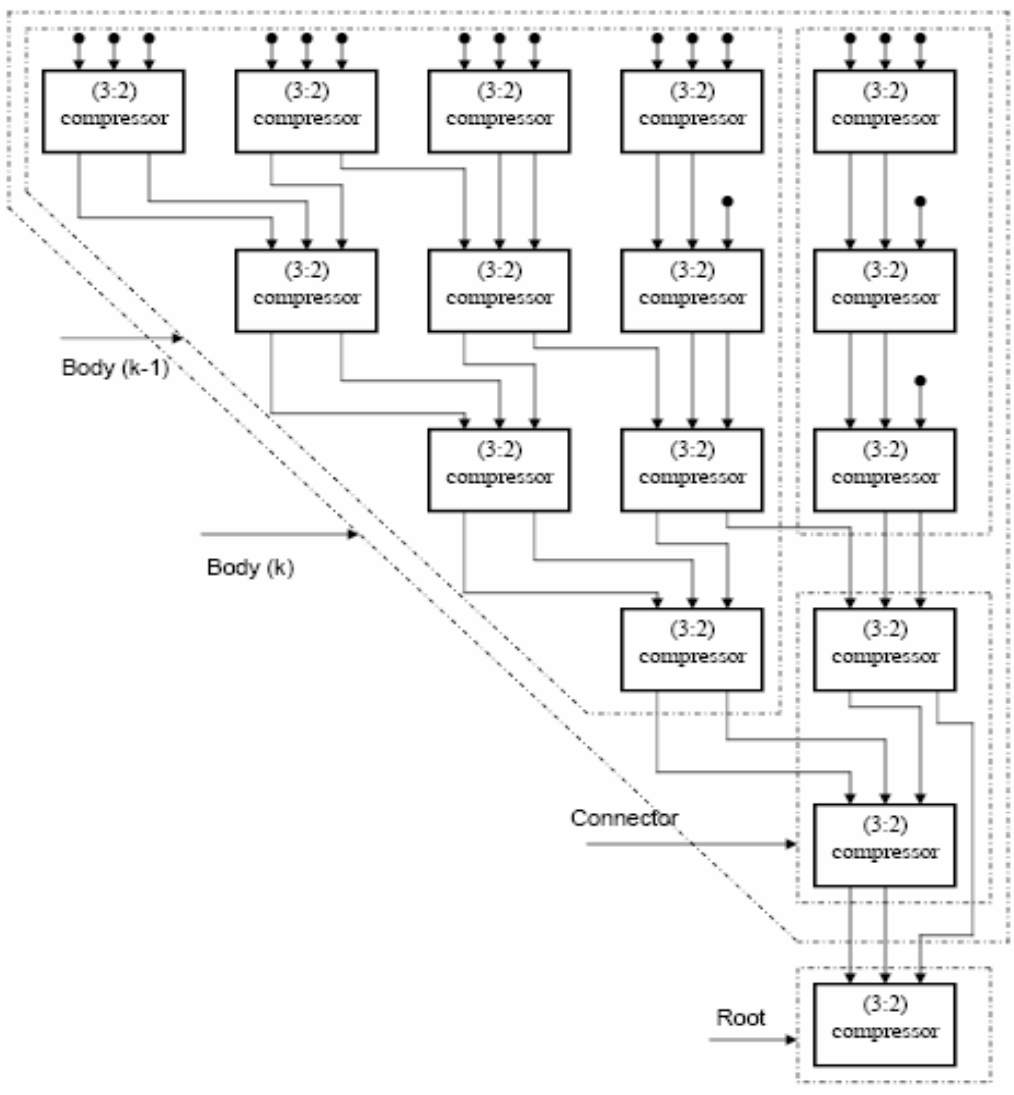

Figure 27: Overturned-staircase tree with 18 PPs

\subsubsection{Wallace Tree}

Wallace trees are irregular in the sense that the informal description does not specify a systematic method for the compressor interconnections. However, it is an efficient implementation of adding partial products in parallel. The Wallace tree operates in three steps:

1. Multiply: Each bit of multiplicand is ANDed with each bit of multiplier yielding $\mathrm{n}^{2}$ results. Depending on the position of the multiplied bits, the wires carry different weights, for example, wire of bit $\mathrm{a}_{2} \mathrm{~b}_{3}$ weighs 32 [16].

2. Addition: As long as there are more than 3 wires with the same weights add a following layer. Take 3 wires of same weight and input them into a full adder. The result will be an output wire of 
same weight. If there are two wires of same weight, add them using half-adder and if only one is left, connect it to the next layer.

3. Group the wires in two numbers and add in a conventional adder.

Wallace tree has been used during the design of multipliers for this thesis. A typical Wallace tree architecture is shown in Figure 28.

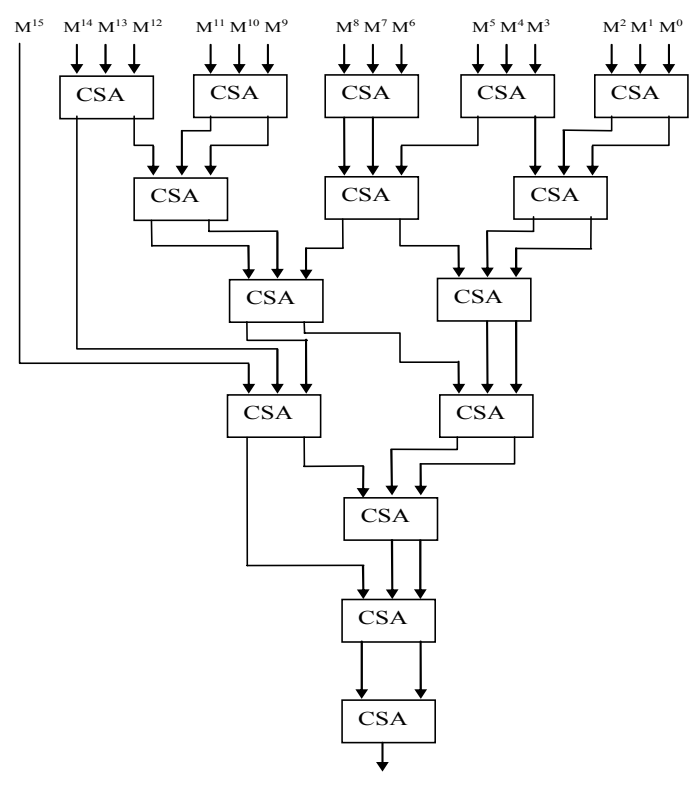

Figure 28: Typical Wallace tree

\subsection{Serial multipliers}

Bit serial arithmetic is a viable alternative in digital signal processing applications to traditional bit-parallel arithmetic. The major advantage of bitserial arithmetic is that it significantly reduces chip area. This is done in two ways; first it eliminates wide buses and simplifies wire routing. Secondly, small processing elements are used which make the chip smaller and require short wiring. A small chip supports higher clock frequencies and is therefore faster [4].

The multiplication of two numbers $\mathbf{a}$ and $\mathbf{x}$ is performed by first generating the partial products by multiplying the coefficient a by every bit of $\mathbf{x}$. The partial products are then added bit-serially with the proper weighting. Most of the bitserial multipliers are based on the shift and add algorithm where several bitproducts are added in each time slot. Bit serial multipliers are classified as 
serial/parallel multiplier, Transposed serial/parallel multiplier and S/P multiplier-accumulator, which will be discussed in the next sections.

\subsubsection{Serial/parallel multiplier}

In a serial/parallel multiplier, the multiplicand $\boldsymbol{x}$ arrives bit-serially while the multiplier $\boldsymbol{a}$ is applied in a bit-parallel format. A common approach used in such multipliers is to generate a row or diagonal of bit-products in each time slot and perform the additions concurrently. Suppose the data is positive $x>0$. Using carry save adders, shift and add algorithm can be applied as shown in Figure 29.

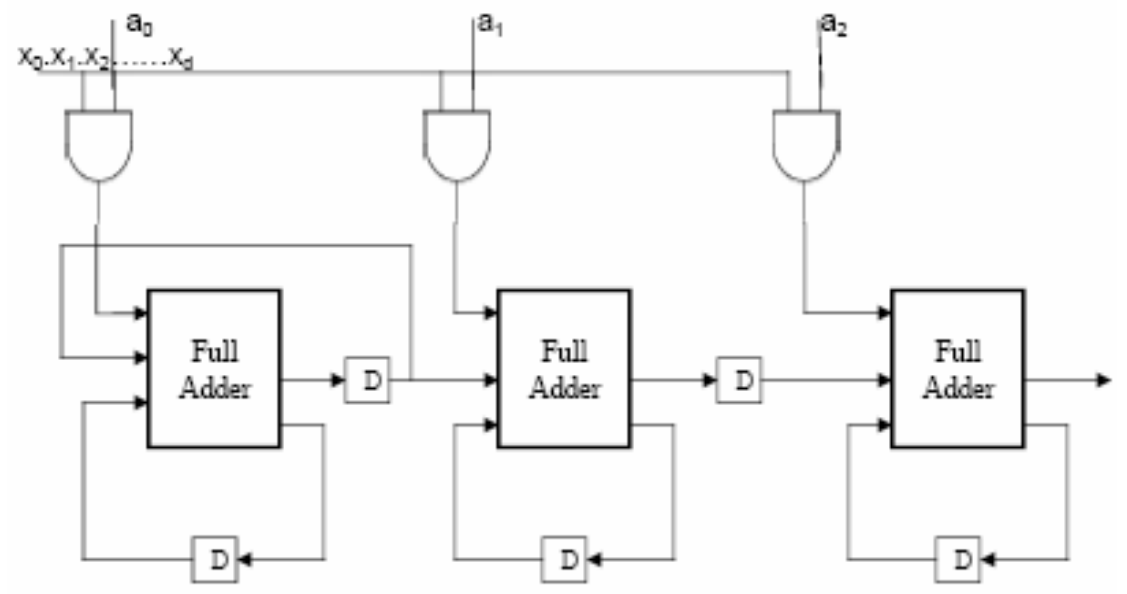

Figure 29: Serial/parallel multiplier

Since $\mathbf{x}$ is processed bit-serially and co-efficient $\boldsymbol{a}$ is processed bit-parallel, this type of multiplier is called a serial/parallel multiplier.

\subsubsection{Transposed serial/parallel multiplier}

This is an alternative form of serial/parallel multiplier, which adds the bitproducts column wise as shown in Figure 30. The disadvantage of this multiplier is a long sum-propagation path. 


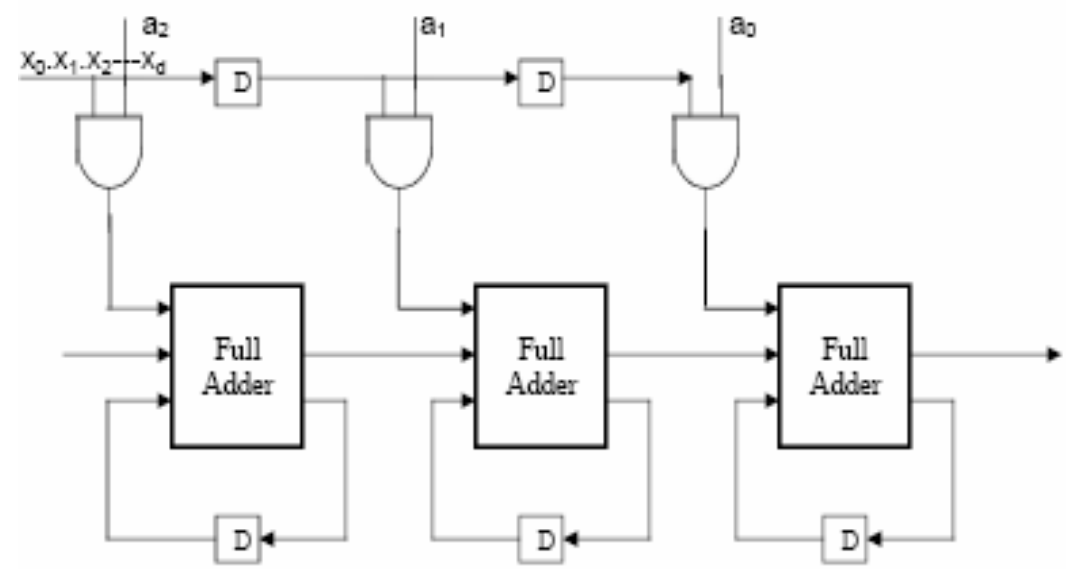

Figure 30: Transposed serial/parallel multiplier

This disadvantage can be alleviated by pipelining, at a cost of two D flip flops per stage [4]. This multiplier structure can be modified into a serial/parallel or squarer where both the multiplier and the multiplicand arrive bit-serially.

\subsection{Serial/Parallel Multiplier Accumulator}

An alternative realization of the serial/parallel multiplier with an additional input that allows computations of the type $y=a . x+z$ is in Figure 31 .

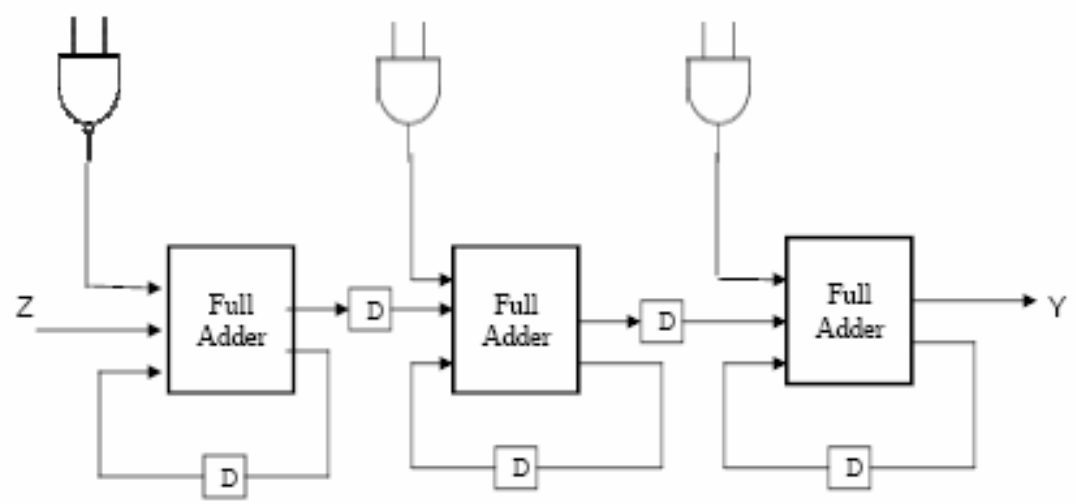

Figure 31: S/P multiplier accumulator

A multiplier-accumulator is obtained if the output $y$ is truncated or rounded to the same word length as $\mathrm{x}$ and added to the subsequent multiplication [4]. 


\section{PART IV}

\section{ENERGY SAVING SCHEMES}


* 


\section{Power consumption issues}

Power consumption is one of the basic parameters of any kind of Integrated Circuit (IC). Power and performance are always traded off to meet the system requirements. Power has a direct impact on system cost. If an IC is consuming more power than a better cooling mechanism would be required to keep the circuit in normal conditions. Otherwise its performance is degraded and on continuous use it may be permanently damaged. The power consumption in CMOS circuits is described below in detail.

\subsection{CMOS power consumption}

There are three types of power consumption in CMOS circuits. Theses are static leakage, short circuit and dynamic switching power consumption. We will discuss these three types considering the example of a simple inverter.

\subsubsection{Static power}

The static or steady state power dissipation of a circuit is expressed by the following relation [1]

$$
P_{\text {stat }}=I_{\text {stat }} V_{d d}
$$

Where $I_{\text {stat }}$ is the current that flows through the circuit when there is no switching activity. Ideally the portion of static current should be zero as the PMOS and NMOS transistors are never on simultaneously in steady-state operation. But unfortunately, there exits a leakage current flowing through the reverse-biased diode junctions of the transistors located between the source/drain and the substrate as shown in Figure 32.

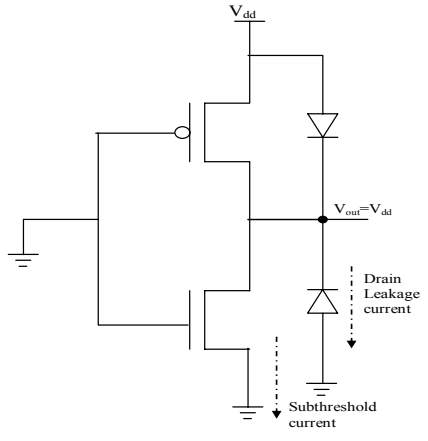

Figure 32: Sources of leakage currents in CMOS [17] 
There are two types of leakage currents: reverse-bias diode leakage on the transistor drains, and sub-threshold leakage through the channel of an "off" device. The magnitude of these currents is set predominantly by the processing technology.

The diode leakage occurs when a transistor is turned off and another active transistor charges up/down the drain with respect to the former's bulk potential. In the case of the inverter with a high input voltage, the output voltage will be low because the NMOS transistor is on. The PMOS transistor will be turned off, but its drain-to-bulk voltage will be equal to the supply voltage $\mathrm{V}_{\mathrm{dd}}$. The resulting diode leakage current will be approximately $I_{1}=A_{d} J_{s}$, where $A_{d}$ is the area of the drain diffusion, and $J_{\mathbf{s}}$ is the leakage current density set by the technology. Since the diode reaches maximum reverse bias current for relatively small reverse bias potential, the leakage current is roughly independent of supply voltage. It is proportional to diffusion area and perimeter. So it is desired to minimize the diffusion area and perimeter in the layout. The leakage current density is temperature sensitive, so $\mathrm{J}_{\mathbf{s}}$ can increase dramatically at higher temperatures [17].

\subsubsection{Short circuit power}

Short circuit currents occur when the rise/fall time at the input of a gate is larger than the output rise/fall time. For the ideal case of a step input, the transistors change state immediately, one turning on and other turning off. There is not a conductive path from the supply to ground. For real circuits, however, the input signal will have some finite rise/fall time. While the condition

$V_{t n} \leq V_{i n} \leq V_{d d}-\left|V_{t p}\right|$ holds for the input voltage, there will be a conductive path open because both devices are on.

The longer the input rise/fall time, the longer the short-circuit current will continue to flow, and the average short-circuit current will increase. To minimize the total average short-circuit current power, it is desirable to have equal input and output edge times.

Short-circuit current power is either linearly or quadratic dependent on the supply voltage, depending on the size of the channel length. While reducing the supply increases the duration of the current linearly due to increased rise/fall times, the peak magnitude of the current is reduced linearly (velocity saturation) such that the average current is approximately constant and the average power is just a linear function of supply voltage $(\mathrm{P}=\mathrm{IV})$. For larger devices that are not 
velocity saturated, the average current is approximately linear with supply voltage so that the average power is a quadratic function of supply voltage [17]. We can compute the average power consumption as follows [1].

$$
P_{s c}=t_{s c} V_{d d} I_{p e a k} f=C_{s c} V_{d d}^{2} f \quad \text { Eq } 4.2
$$

$t_{s c}$ represents the time both devices are conducting.

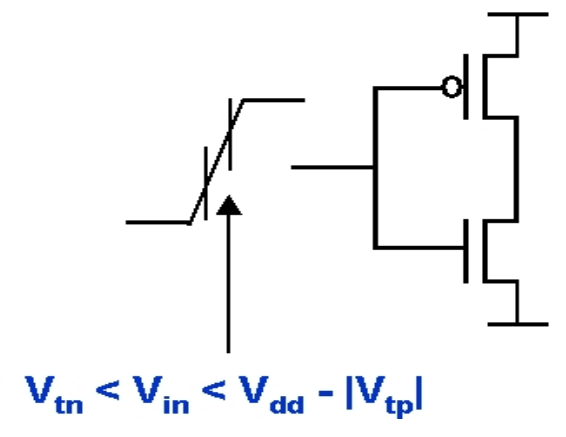

Figure 33: Short circuit current in CMOS circuits

For most ICs, the short-circuit power dissipated is approximately $5-10 \%$ of the total dynamic power. If the supply is lowered to below the sum of the thresholds of the transistors i.e. $V_{d d}<V_{t n}+V_{t p}$. However, short-circuit currents will be eliminated because both devices cannot be on at the same time for all values of input voltage.

\subsubsection{Dynamic switching power}

This is a major component of CMOS power consumption. This power exists when the output capacitance $\mathrm{C}_{\mathrm{L}}$ charges through PMOS and discharges through NMOS transistor. Each time the capacitor $\mathrm{C}_{\mathrm{L}}$ gets charged through the PMOS transistor, its voltage rises from 0 to $\mathrm{Vdd}$ and a certain amount of energy is drawn from the power supply. Part of this energy is dissipated in the PMOS device while the remaining energy is stored on the load capacitance. During the high-to-low transition, this capacitor is discharged and the stored energy is dissipated in the NMOS transistor as shown in Figure 34. 


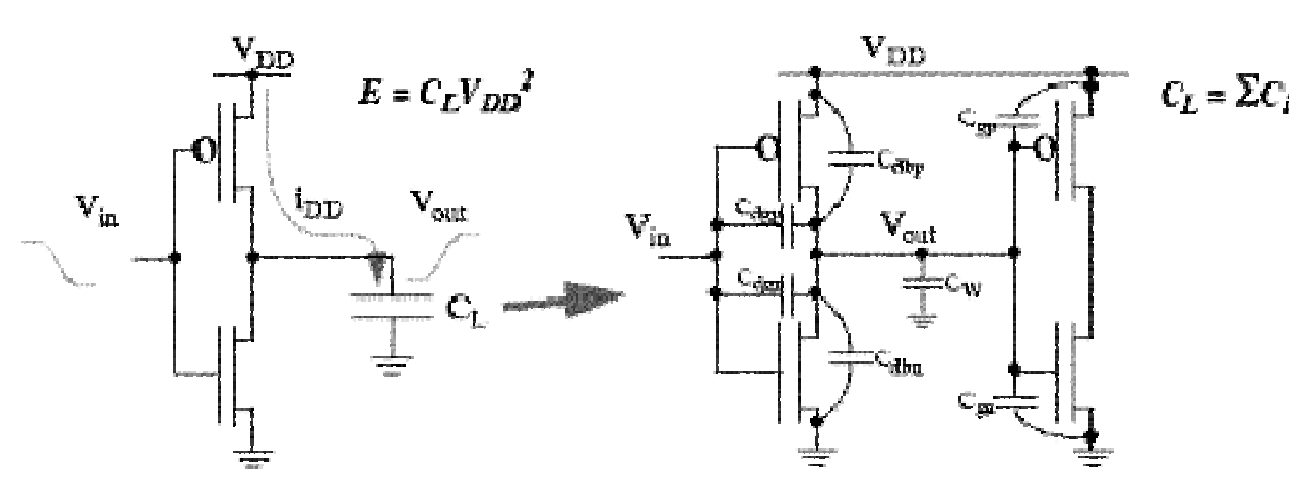

Figure 34: Equivalent circuit for load capacitance [18]

The exact formula for dynamic power consumption is as follows

$$
P_{d y n}=C_{L} V_{d d}^{2} f_{0 \rightarrow 1}
$$

Where $f_{0 \rightarrow 1}$ represents the frequency of energy-consuming transitions. This factor is also called the switching activity.

\subsection{Low power techniques}

Power consumption becomes an issue in complex electronic systems where cost is extremely important. Reducing power consumption is an important design task for IC engineers. Power is important for portable equipment like mobile, laptop, PDA, GPS, hearing aids and wrist watch etc. The requirement is a long life battery and a light system which is only possible if the equipment consumes bare minimum power. Lowering power also reduces the cost for cooling system and makes the chip package smart reducing the size of the device.

There are a number of low power techniques available for CMOS circuits. Some of them are discussed in subsequent sections.

\subsubsection{Delay balancing}

Glitches in the circuit consume a considerable amount of power. They are produced by a delay in the arrival of input signals at a certain gate in the circuit. Delay balancing is then used to minimize the glitches which in return saves power. Suppose we want to add four inputs A, B, C and D. One way to add them is to feed the inputs sequentially as shown in Figure 35 (a). Suppose the delay of one adder is $T_{\text {add }}$. The inputs $A$ and $B$ arrive at the same time at adder 1 but there exits a delay of $1 \mathrm{~T}_{\text {add }}$ and $2 \mathrm{~T}_{\text {add }}$ between the inputs arriving at adder 2 
and 3 respectively. This would produce glitches at the output node. In order to remove glitches, we have to balance the delays at the inputs of each adder. Figure 35 (b) shows a modified architecture in which the delays are balanced at the input of each adder.

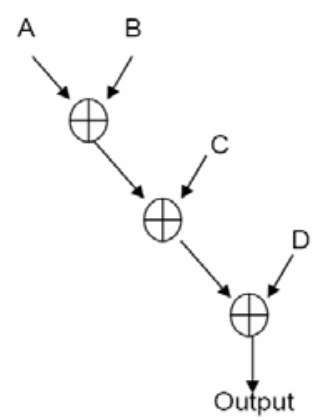

(a)

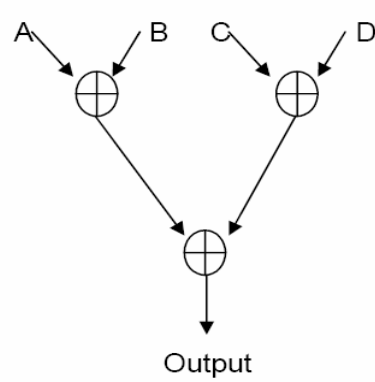

(b)

Figure 35: Delay balancing example

\subsubsection{Clock gating}

Clock gating is one of the power-saving techniques used on many synchronous circuits including the Pentium 4 processor. To save power, clock gating refers to adding additional logic to a circuit to prune the clock tree, thus disabling portions of the circuitry where flip flops do not change state [19].

Assume a pipelined system to compare two numbers as shown in Figure 36. The first stage is a combinational logic block while the second stage is a comparator which performs $\mathrm{A}>\mathrm{B}$ where $\mathrm{A}$ and $\mathrm{B}$ are generated in the first stage. If the MSBs A [N-1] and B [N-1] are different, then the computation can be performed strictly from the MSBs and therefore the comparator logic for $A[\mathrm{~N}-2$ : $0]$ and $\mathrm{B}[\mathrm{N}-2: 0]$ can be avoided for power saving. 


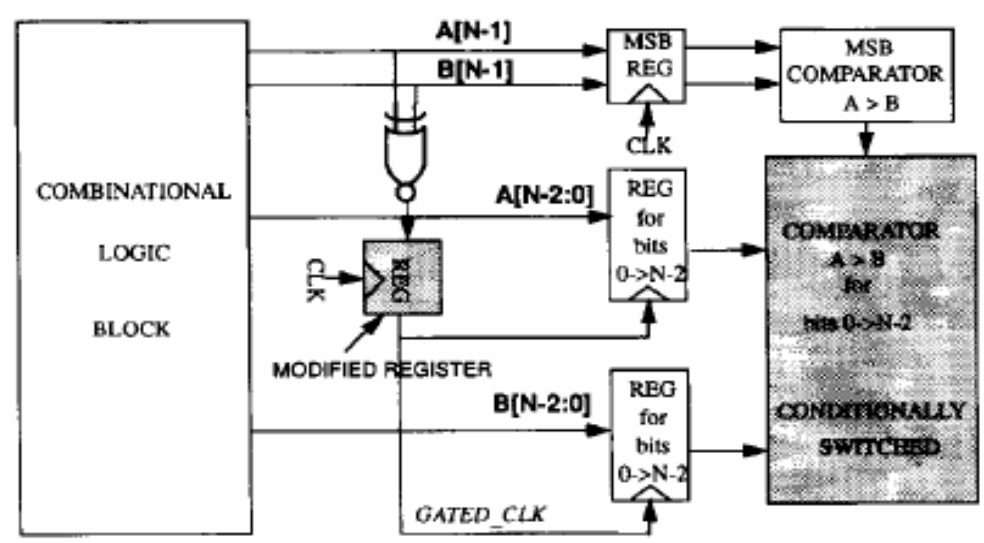

Figure 36: Clock gating technique for power saving

One approach to accomplish this is to gate the clock as shown in Figure 36. The XNOR output of $A[\mathrm{~N}-1]$ and $\mathrm{B}[\mathrm{N}-1]$ is latched by a register to generate a gated clock [6]. This gated clock is then used to clock the lower order registers.

\subsubsection{Voltage scaling}

In CMOS circuits, the dominant component of power consumption is proportional to $\mathrm{V}_{\mathrm{dd}}^{2} \mathrm{f}$, where $\mathrm{V}_{\mathrm{dd}}$ is voltage and $\mathrm{f}$ is frequency. Energy is product of power and time. The time to run a certain number of cycles is inversely proportional to frequency, so energy per cycle is proportional to $\mathrm{V}_{\mathrm{dd}}{ }^{2}$. At a given voltage, the maximum frequency at which the circuit can run safely decreases with decreasing voltage. Thus, the system can reduce energy consumption by reducing supply voltage, but this necessitates running at a slower speed [20].

\subsubsection{Transition activity reduction}

The power consumption is directly proportional to the transition or switching activity of the circuit. So reducing transition activity will also reduce the power consumption of the circuit. To reduce the transition activity in complex electronic systems, some special encodings are used for data and address busses. These encodings are very effective in reducing the power consumption. These are Limited Weight Codes (LWC), Zero-transition encoding (T0), Bus-invert encoding (BI), TO_BI encoding, Dual_T0 encoding etc. an example of Businvert encoding is shown in Table 3.

In bus-invert encoding, an extra line is used. If the extra bit is zero, the original bits are kept intact and they are inverted if the extra bit is 1 . The overhead is one 
extra bit line but the number of transitions is reduced significantly which in turn reduces the power consumption.

\begin{tabular}{|c|c|c|c|}
\hline Input sequence & $\begin{array}{c}\text { Number of } \\
\text { Transitions }\end{array}$ & $\begin{array}{c}\text { Bus_invert } \\
\text { Encoded } \\
\text { sequence }\end{array}$ & $\begin{array}{c}\text { New number of } \\
\text { transitions }\end{array}$ \\
\hline 0000 & 1 & 00000 & 1 \\
\hline 0001 & 1 & 00001 & 1 \\
\hline 0010 & 2 & 00010 & 2 \\
\hline 0011 & 1 & 00011 & 2 \\
\hline 0100 & 3 & 11011 & 1 \\
\hline 0101 & 1 & 11010 & 2 \\
\hline 0110 & 2 & 11001 & 1 \\
\hline 0111 & 1 & 11000 & 12 \\
\hline 1000 & 4 & 01000 & $\mathbf{1 2}$ \\
\hline Total transitions & $\mathbf{1 6}$ & & \\
\hline
\end{tabular}

Table 3: Bus invert encoding

\subsubsection{Pipelining}

Pipelining is a technique used along with voltage scaling to reduce the power consumption. The idea is to insert registers after some appropriate distance in the circuit. The system response becomes faster than before. In order to maintain the previous delay, the supply voltage is reduced which reduces the power consumption. Assume we have to perform multiplication operation $\mathrm{X} 1 . \mathrm{X} 2 . \mathrm{X} 3 . \mathrm{X} 4$. The delay of one multiplier is $\mathrm{T}_{\mathrm{mul}}$ at a supply voltage is $\mathrm{V}_{0}$. The basic architecture is shown in Figure 37 (a).

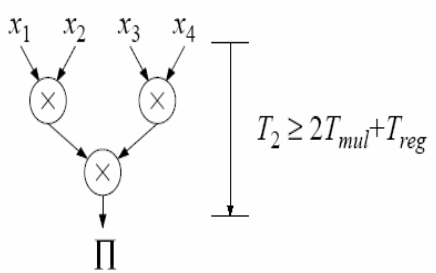

(a)

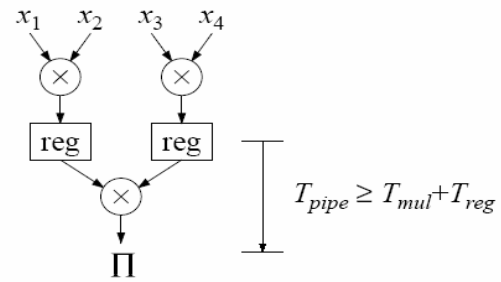

(b)

Figure 37: Pipelining example

Now we pipeline the tow multipliers with two additional registers. The new architecture is as shown in Figure 37 (b). For this case, the additional throughput can be traded for lower power dissipation if the overhead of the two registers is less than the gain of supply voltage scaling. 


\subsubsection{Interleaving}

In this technique, the circuit components are duplicated and the frequency of operation is halved for both portions of the circuit. One half operates on positive clock edge and the other operates on the negative clock edge. A multiplexer is used to select the result one by one from both portions of the circuit. The throughput of the system is doubled by this way, which provides a time margin. This time margin can be used for supply voltage reduction to reduce power consumption.

An interleaved circuit with identical two blocks using four additional registers and one multiplexer is shown in Figure 38.

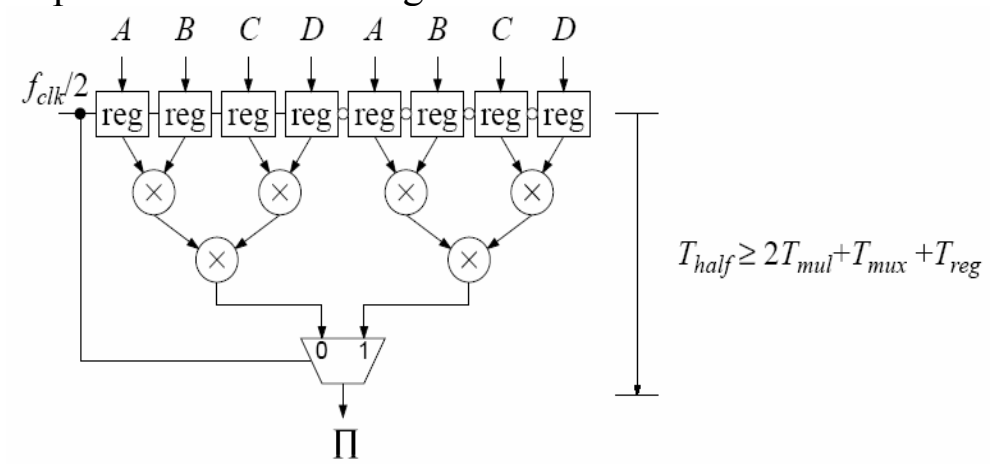

Figure 38: Interleaved circuit for multiplication

Note that the overhead in terms of power of the registers is less than normal registers since they should be clocked at half frequency. The minimal clock frequency in the circuit path is $T_{\text {half }}>2 T_{\text {mul }}+T_{m u x}+T_{\text {reg. }}$. However, since the requirement on throughput in each block is halved, the required clock period is $T_{\text {int }}>T_{\text {mul }}+\left(T_{\text {mux }}+T_{\text {reg }}\right) / 2$, which in many cases should be less than the original $T_{c l k}$. For the interleaved case, the additional throughput can be traded for lower power dissipation if the overhead of the half speed registers and the multiplexer is less than the gain obtained with voltage scaling [21].

Often, interleaving and pipelining techniques are used in combination. This results in more power reduction than in the cases where both are used alone. 


\section{PART V}

\section{PROPOSED ARCHITECTURE}


* 


\section{Energy-efficient $\mathbf{3 2}$ bit multiplier architecture}

The previous sections provided a background about multiplier architectures, types, power consumption issues and methods to reduce the power. This section describes the real thesis work performed for the development and evaluation of energy-efficient fast 32 bit multiplier architecture.

\subsection{Previous related work}

There are a number of multiplier architectures presented by the researchers during the last decade. A built-in self test (BIST) module is proposed by [7]. In reference [8] a new technique is presented to reduce the partial products. Similarly reference [9] emphasized on using latches to synchronize the inputs to the adder block in order to eliminate spurious switching. A scalable and iterative architecture for pipelined Wallace tree multipliers is described in [10]. The final adder must be fast enough to compute the results. A carry select adder is a suitable choice for that. A carry select adder optimization technique is presented in [11].

A well defined and novel approach was presented by Jung-Yup Kang and JeanLuc Gaudiot in [5]. This paper describes a scheme to eliminate the sign extension problem produced in MBE Wallace multipliers. The present thesis work is based upon this architecture. They have proved that taking the 2's complement of the multiplicand reduces one row of partial products and a negative signal, which reduces the carry save adder tree (CSAT). The reduction of CSAT also reduces the delay and power consumption. In this paper they also presented a fast technique to compute the 2 's complement of a binary number.

\subsection{Architecture description}

The new architecture proposed by [5] consists of Partial Product Generation (PPG) logic, 2's complement logic and a carry save adder tree as described in section 4. The concentration is on the first step which consists in forming the partial product array and designs a multiplication algorithm which will produce fewer partial product rows. By having fewer partial product rows, the reduction tree can be smaller in size and faster in speed. 


\subsubsection{Multiplication Algorithm}

There is no doubt that MBE is efficient in reducing the partial products. However, it is important to note that there are two unavoidable consequences when using MBE: sign extension prevention and negative encoding.

The combination of these two unavoidable consequences results in the formation of one additional partial product row and of course, this additional partial product row requires more hardware but more importantly time [5].

The grouping of the multiplier bits of MBE is shown in Figure 4 and it is based on a window size of 3 bits and a stride of 2 . The multiplier $(\mathrm{Y})$ is segmented into groups of three bits $\left(\mathrm{y}_{2 \mathrm{i}+1}, \mathrm{y}_{2 \mathrm{i}}, \mathrm{y}_{2 \mathrm{i}-1}\right)$ and each such group of bits is associated with its own partial product row by using Table 2. By applying this encoding, the number of partial product rows to be accumulated is reduced from $\mathrm{n}$ to $\mathrm{n} / 2$. However, there are actually $\mathrm{n} / 2+1$ partial product rows rather than $\mathrm{n} / 2$, because of the last neg signal as shown in Figure 39. Consequently, one additional carry save adding stage is needed to perform the reduction. This is the overhead of implementing the negative encodings of MBE.

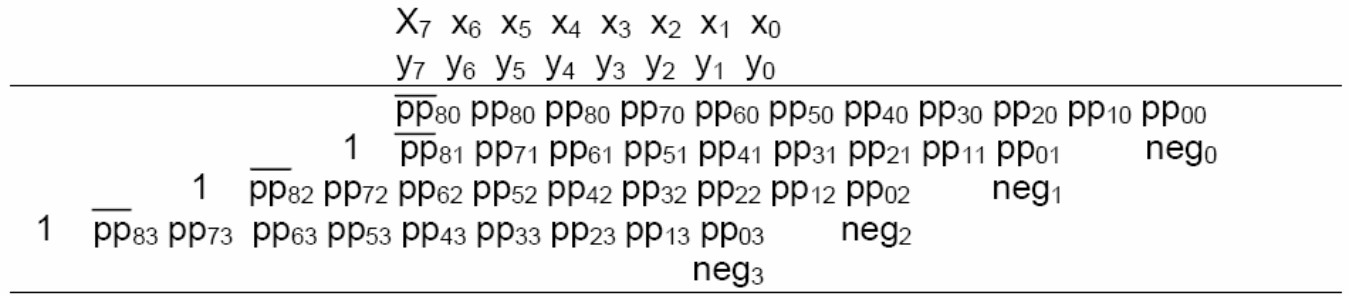

Figure 39: Multiplication algorithm with negative encoding

\subsubsection{Sign extension problem and solution}

In signed multiplication, the sign bit of a partial product row would have to be extended all the way to the MSB position which would require the sign bit to drive that many output loads. This makes the partial product rows unequal in length as shown in Figure 40. The first row spans 16 bits (pp00 to the leftmost pp80), the second row 14 bits (pp01 to the leftmost pp81), the third row 12 bits (pp02 to the leftmost pp82), and the fourth row 10 bits (pp03 to the leftmost pp83). Ercegovac [12] has shown sign extension prevention method and arrives at a newly formed partial product rows as in Figure 44 where the sign extension has been removed. This is the basic structure for our new multiplier. 


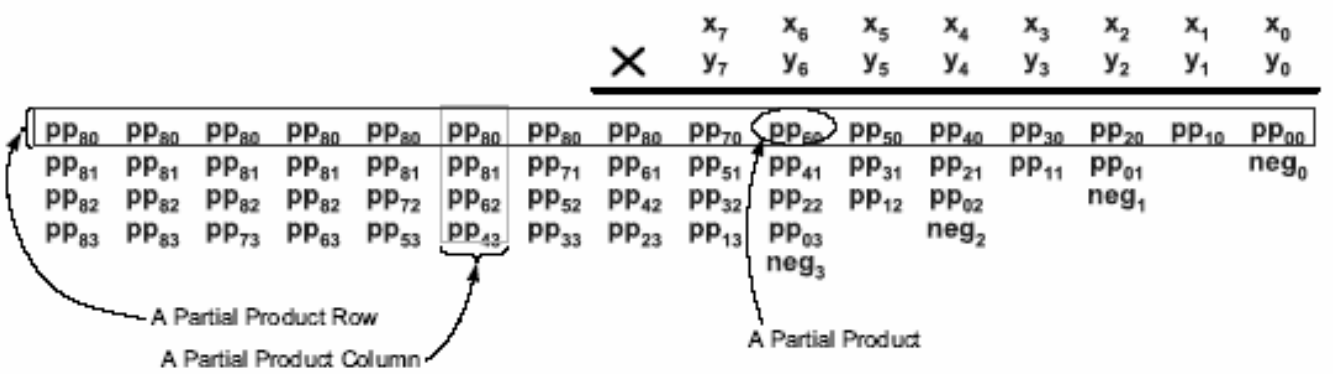

Figure 40: Multiplication with sign extension [5]

\subsubsection{Fast method for 2's complement}

The final neg signal in Figure 40 gives rise to one additional partial product row which means one additional 3-2 reduction in CSAT. This negative signal can be eliminated completely if we take the 2's complement of the multiplicand while the other partial products are produced in a normal way. By this way there will not be any neg signal in the last PPR and hence the need for an extra CSA is diminished. Therefore, we only need to find a faster method to calculate the 2's complement of a binary number.

Two's complementation complements all the bits after the rightmost " 1 " in the word but keeps the other bits as they are. The two's complement of a binary number $001010_{2}(1010)$ is $110110_{2}(-1010)$ as shown in Figure 41.

For this number, the rightmost "1" happens in bit position 1 (the check mark position in Figure 41). Therefore, values in bit positions 2 to 5 can simply be complemented while values in bit positions 0 and 1 are kept as they were. Therefore, two's complementation now comes down to finding the conversion signals that are used for selectively complementing some of the input bits [5].

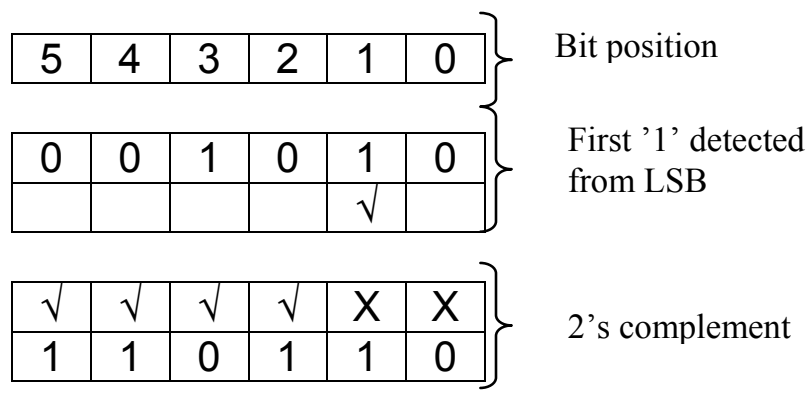

Figure 41: Two's complement conversion 
If the conversion signal at any position is " 0 " then the value is kept as it is and if it is " 1 ", then, the value is complemented.

However, the search for the rightmost " 1 " is a time consuming task and it can be done in algorithmic time using a binary search tree-like structure. The method starts by first finding the conversion signals for two consecutive 2-bits starting from the LSB position and finding the conversion signals for each group. Then find the conversion signals for a 4-bit group, then for 8-bits and so on until the whole input bits are covered. The method is shown below in Figure 42 .

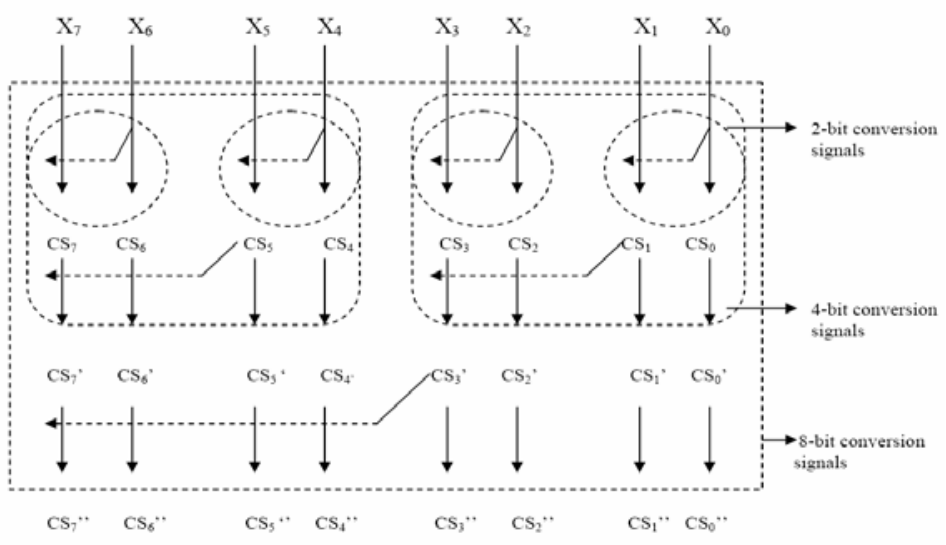

Figure 42: Finding conversion signals for 8 bit number

Based upon the above mentioned technique Jung-Yup and Gaudiot developed the following logic design for computing 2's complement.

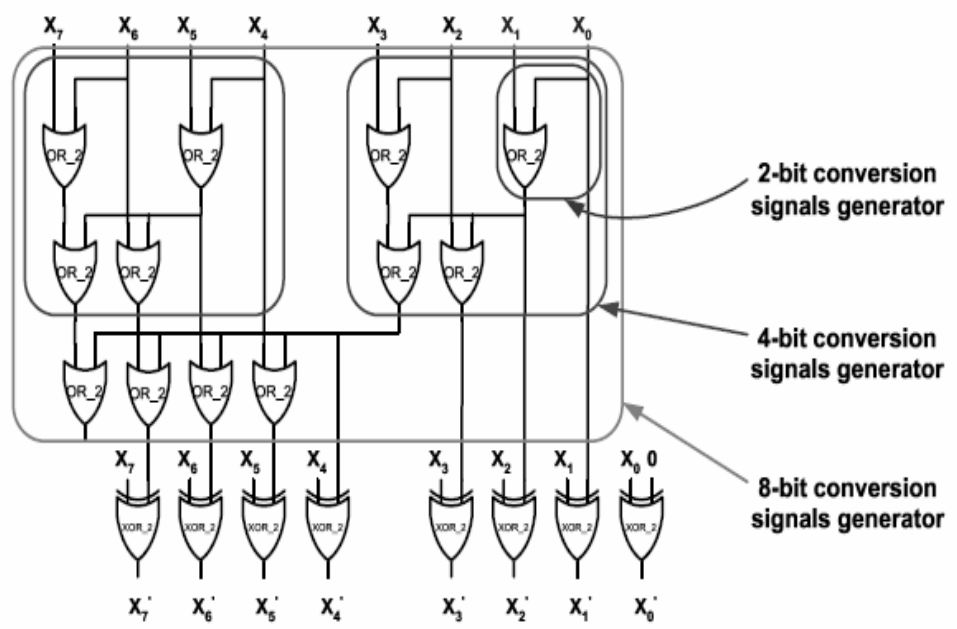

Figure 43: Fast logic for 2's complement computation [5] 


\subsection{Development of full architecture}

In order to develop the full multiplier architecture, following steps have to be followed.

1. The last partial product row is replaced with signals $\mathrm{S}_{0} \sim \mathrm{S}_{32}$.

2. A series of $1 \mathrm{~s}$ is appended at the end of the second last row to restore it back to the original sign extended format.

3. The MSB of the last row is complemented and the 1 directly above it is removed.

After completing the above three steps, the new algorithm looks like as shown in Figure 44.

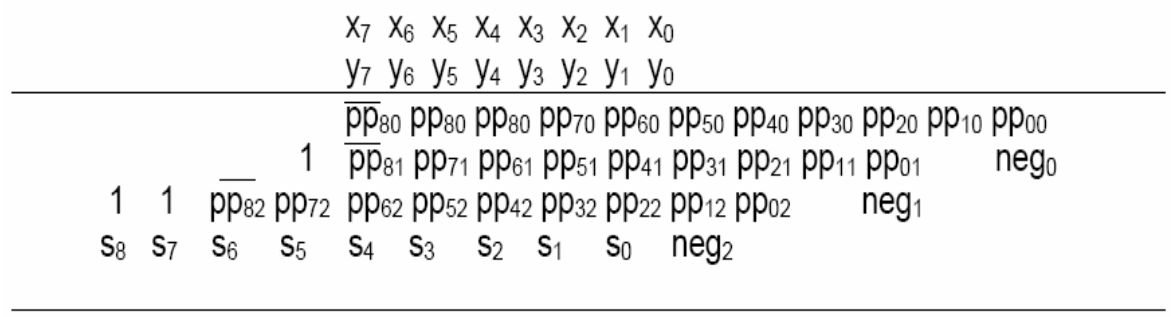

Figure 44: New proposed algorithm

The signals $\mathrm{s}_{0} \sim \mathrm{s}_{9}$ are obtained from the last partial product generator row, the output of 5-1 selector. The neg signals can be directly obtained from MBE table. This signal is " 0 " for the first four entries the table and "1" for the last four. The new architecture is shown in Figure 45. 


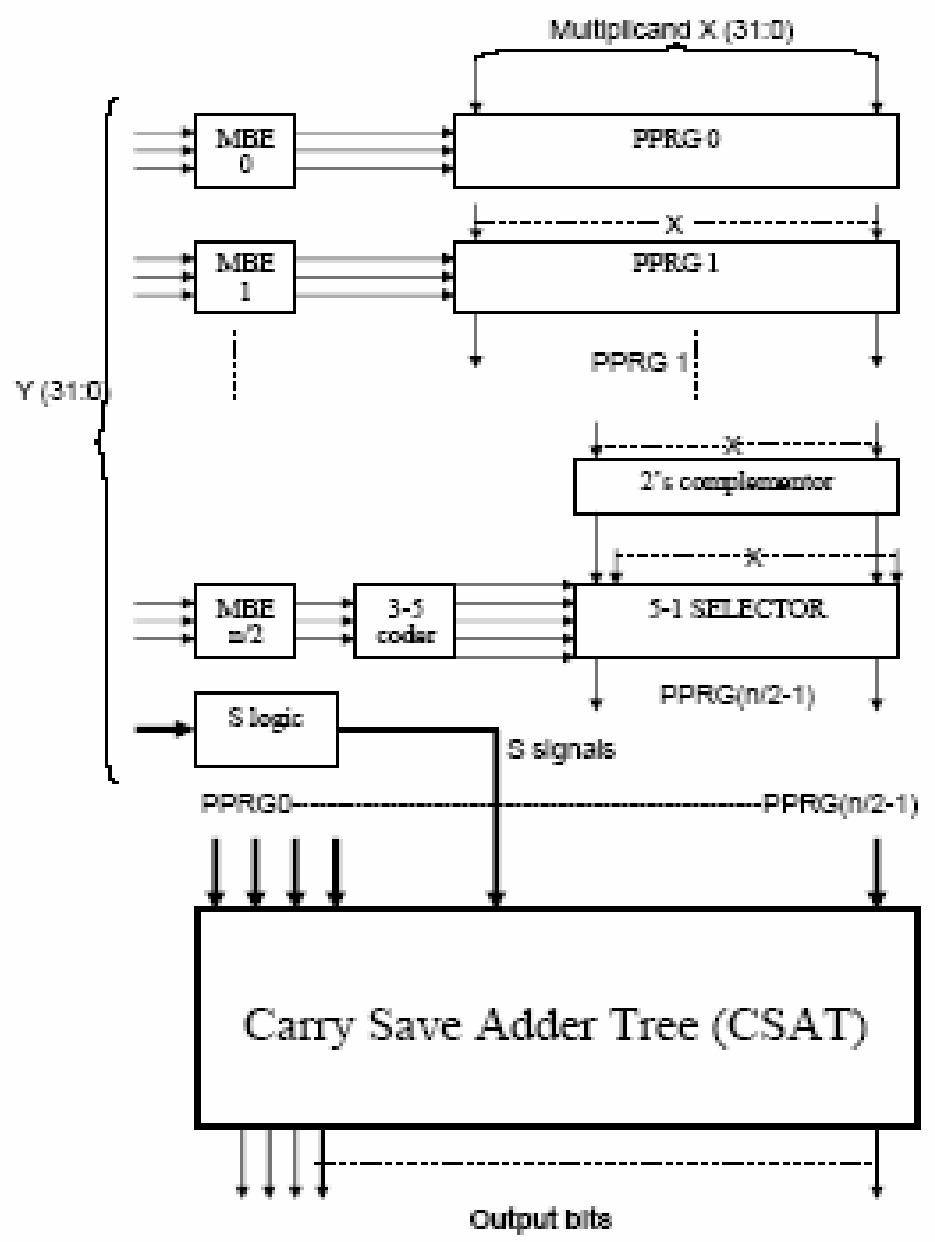

Figure 45: New proposed multiplier architecture

The new multiplier architecture saves one CSA by eliminating the last neg signal as discussed in the previous sections.

The logic design for MBE can be obtained from Table 2 by generating output signals one, two and neg. the designs of all other components are proposed by [2] and are shown in the sub sequent figures. 


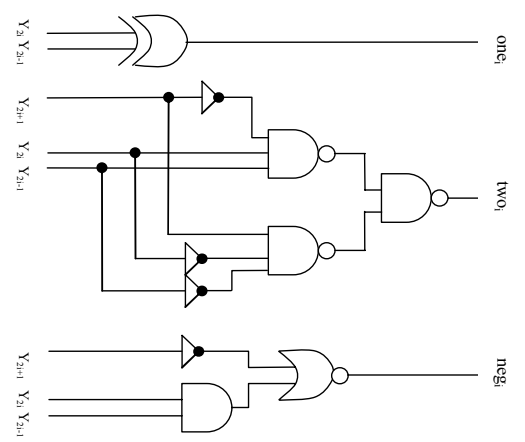

Figure 46: MBE logic

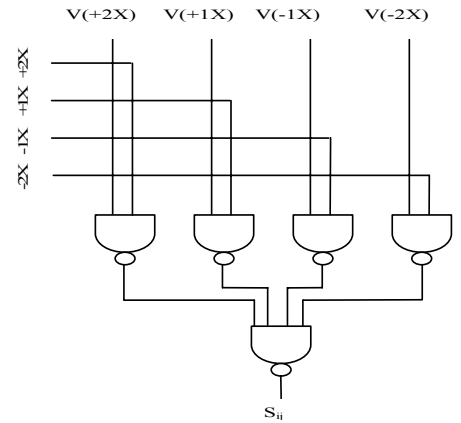

Figure 47: 5-1 selector

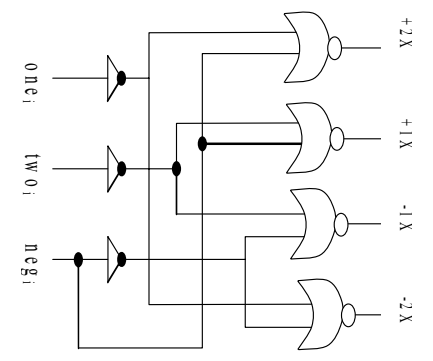

Figure 48: 3-5 decoder

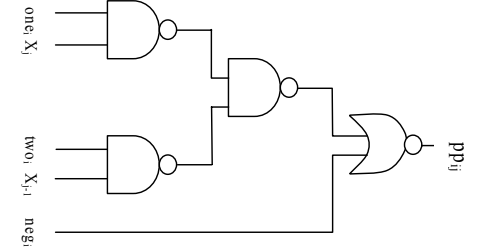

Figure 49: Partial product generation 


\subsection{Critical path calculations}

Critical path is the longest path in a circuit from input to output. Critical path (CP) calculation is very important while estimating the delay of the circuit under test. CP for conventional multiplier consists of one PPG in series with a chain of 9 Full Adders (FA). The PPG itself consists of a simple AND gate.

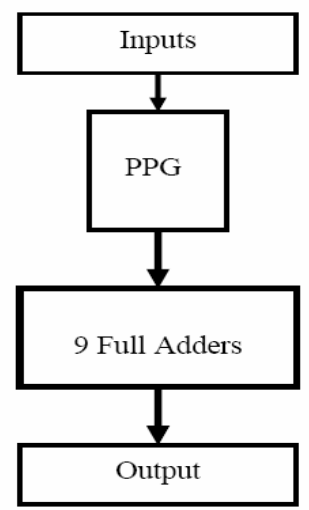

Figure 50: Critical path of conventional multiplier

For simplicity it is assumed that the delay of an inverter as unity, so that the delays of all logic gates can be described in terms of inverter delays. Based on this assumption, the delays of various gates are calculated in Table 4 .

\begin{tabular}{|c|c|}
\hline Module & Delay \\
\hline Inverter & 1 \\
\hline NAND2 & 2 \\
\hline NAND3 & 3 \\
\hline NAND4 & 4 \\
\hline NOR2 & 2 \\
\hline XOR & 3 \\
\hline AND2 & 3 \\
\hline FA (2 XOR) & 6 \\
\hline
\end{tabular}

Table 4: Normalized delays of gates w.r.t inverter

The critical path delay of conventional multiplier comes out to be

$$
\mathrm{T}_{\mathrm{AND}}+9 \mathrm{~T}_{\mathrm{FA}}=3+9 \mathrm{x} 6=57 \text { units }
$$

$\mathrm{CP}$ for selected multiplier consists of two branches. One path starts from MBE, passes through PPG and then through 8 Full Adders. The second also starts from MBE and then passes through 3-5 coder, 4-1 selector and then 8 FAs as shown below in Figure 51. 


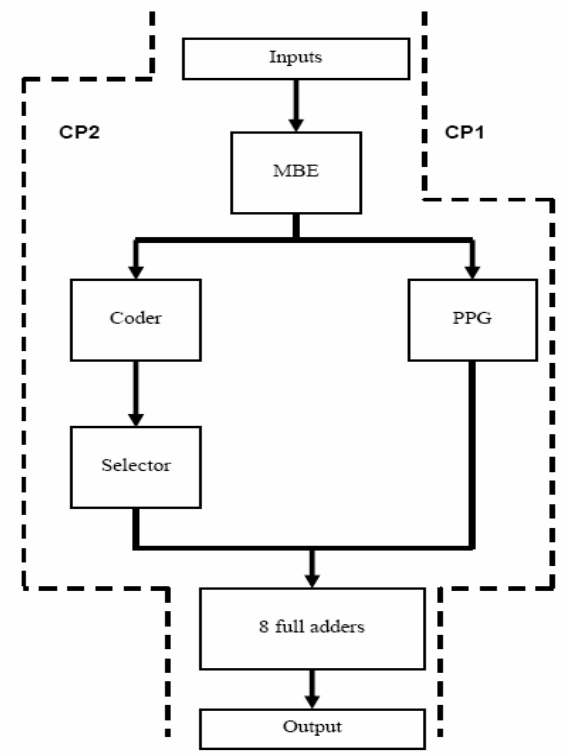

Figure 51: Critical path of new architecture

$$
\begin{gathered}
\mathrm{T}_{\mathrm{CP} 1}=\mathrm{T}_{\mathrm{INV}}+\mathrm{T}_{\mathrm{NAND} 3}+\mathrm{T}_{\mathrm{NAND} 2}+\mathrm{T}_{\mathrm{NAND} 2}+\mathrm{T}_{\mathrm{NAND} 2}+\mathrm{T}_{\mathrm{XOR}}+8 \mathrm{~T}_{\mathrm{FA}} \\
=1+3+2+2+2+3+8 \mathrm{X} 6=61 \text { units } \\
\mathrm{T}_{\mathrm{CP} 2}=\mathrm{T}_{\mathrm{INV}}+\mathrm{T}_{\mathrm{NAND} 3}+\mathrm{T}_{\mathrm{NAND} 2}+\mathrm{T}_{\mathrm{INV}}+\mathrm{T}_{\mathrm{NOR} 2}+\mathrm{T}_{\mathrm{NAND} 2}+\mathrm{T}_{\mathrm{NAND}} 4+8 \mathrm{~T}_{\mathrm{FA}} \\
=1+3+2+1+2+2+4+8 \mathrm{X} 6=63 \text { units }
\end{gathered}
$$

The analysis shows that $\mathrm{CP} 2$ is the longest critical path with a total delay of 63 time units. So we can expect this multiplier to be slower than the conventional one at nominal supply voltage. 
* 


\section{PART VI}

\section{SIMULATION RESULTS}


* 


\section{Simulation results in Cadence ${ }^{\circledR} 90 \mathrm{~nm}$}

Both the multipliers have been simulated using a common test setup in Cadence $\mathbb{R}$. A variety of test vectors are applied at the input ports and the resulting outputs are analyzed using Spectre waveform window. The power consumption and delay is measured in that window. The power is calculated by finding the average current and multiplying it with the supply voltage.

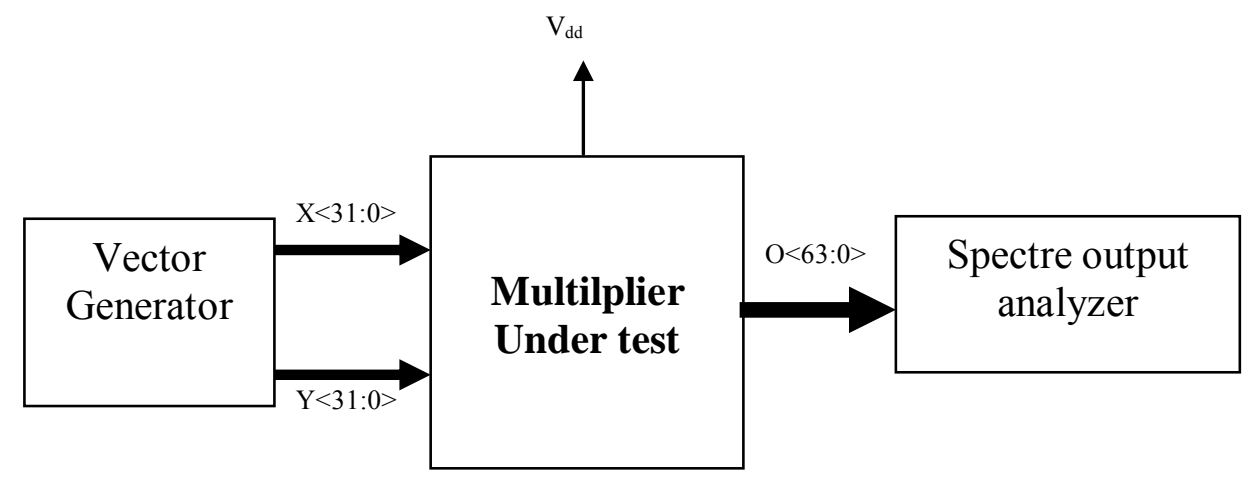

Figure 52: Simulation setup

The nominal voltage for $90 \mathrm{~nm}$ technology is $1.0 \mathrm{~V}$. However, I have also tested the multipliers at $1.2,0.9,0.8$ and $0.6 \mathrm{~V}$ in order to plot the power- delay product.

The functionality of multipliers is tested by first applying all " 0 " signals at the input ports. And then by setting one of the inputs high while keeping the other still " 0 ". And finally, by setting all of the inputs high and measuring the delay and power consumption. The worst case power is obtained when all the inputs are pulled high.

\subsection{Power, performance comparison}

The two multiplier architectures have been simulated using the circuit simulator Spectre and process data from a commercially available 90-nm CMOS technology. A Common test bench has been set up, where the power and performance have been evaluated. Figure 53 shows the power-delay plot of the simulation results. 


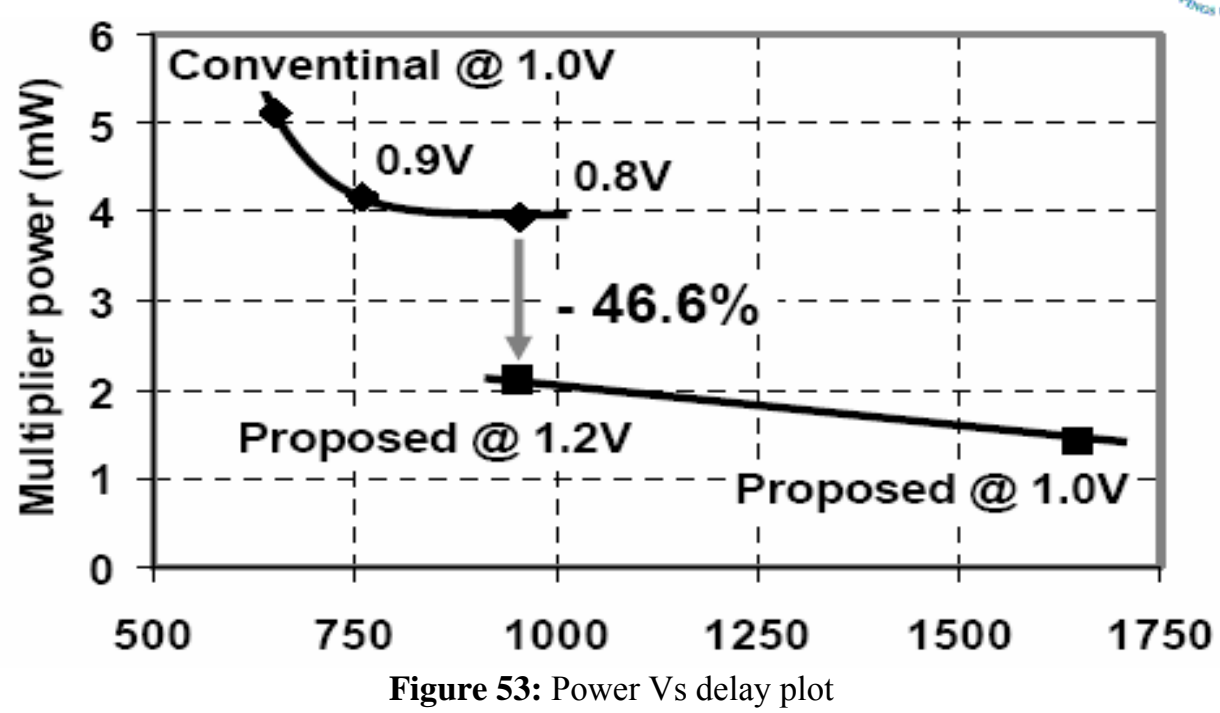

The conventional multiplier has a delay of 652 ps with $5.11 \mathrm{~mW}$ power dissipation at nominal power supply $(1.0 \mathrm{~V})$. The proposed structure with its more complex PPG resulted in 1648ps delay and $1.41 \mathrm{~mW}$ power dissipation at nominal supply. Calculating the power delay-product (PDP) for the two architectures at nominal supply gives $3.33 \mathrm{pJ}$ and $2.32 \mathrm{pJ}$ for the conventional and proposed, respectively. In order to get a fair power comparison of the two multiplier architectures, the power supply of the conventional structure was reduced, while the same for the proposed multiplier was increased until equal latency was achieved. For the same delay, the PDP point of the conventional multiplier was $3.78 \mathrm{pJ}$ compared to $2.01 \mathrm{pJ}$ for the proposed architecture. Hence, the proposed architecture enables $47 \%$ better energy-efficiency for equal throughput and latency.

The main benefit with the MBE architecture is the reduced number of generated partial products. This reduces the amount of combinatorial logic needed to merge partial products to the final result. The conventional architecture required a total transistor count of 58030 transistors. Although the partial product merging tree was reduced by roughly half for the proposed architecture, the additional hardware required for the MBE and 2's complement generation added to the total transistor count. Therefore, the total transistor count of the proposed architecture was 38510 , which still is $34 \%$ less than the conventional. 


\subsection{Chip area comparison}

In order to analyze the chip area and effects of wiring, we have prepared the layouts in $90 \mathrm{~nm}$ CMOS technology of both multipliers. These are shown in Figure 54.

Conventional

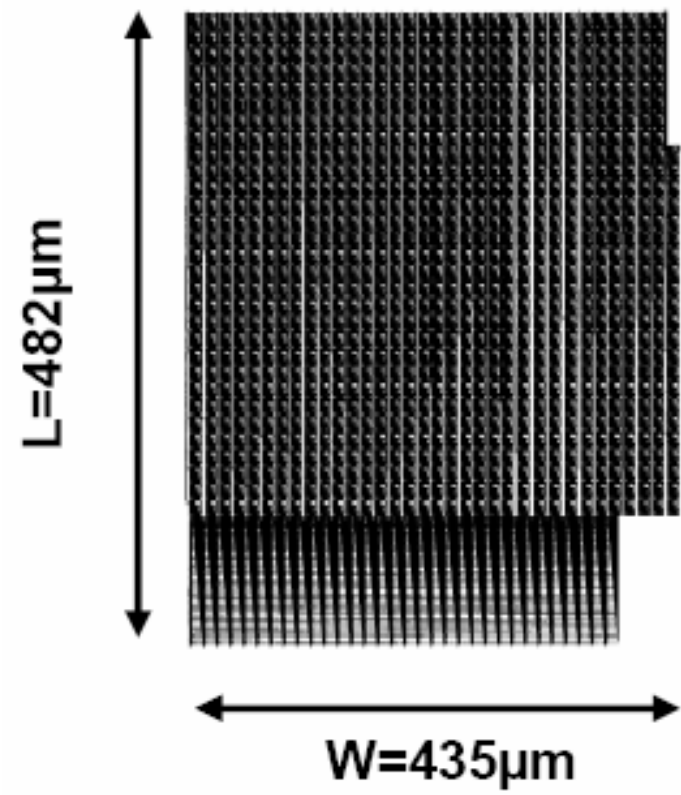

Proposed

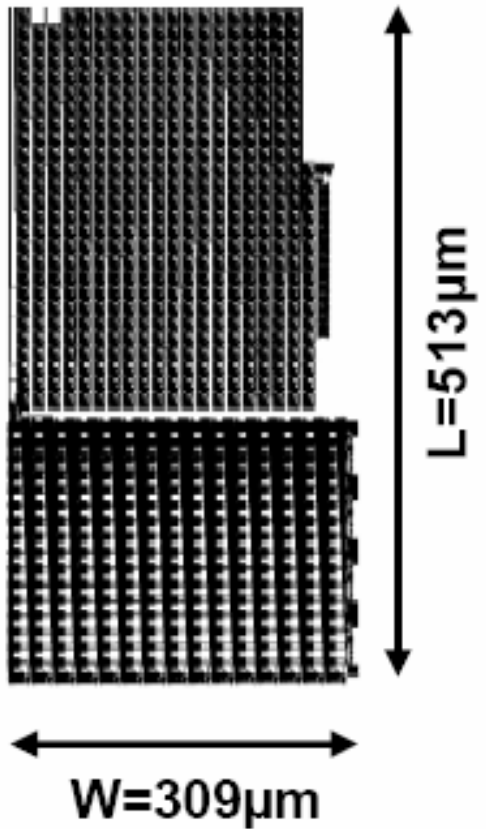

Figure 54: Chip area comparison

Conventional multiplier:

$$
\begin{aligned}
& \mathrm{L}_{\mathrm{c}}=482 \mu \mathrm{m} \\
& \mathrm{W}_{\mathrm{c}}=435 \mu \mathrm{m} \\
& \text { Area }=482 \times 435=209834 \mu \mathrm{m}^{2}=0.21 \mathrm{~mm}^{2} .
\end{aligned}
$$

Proposed multiplier:

$$
\begin{aligned}
& \mathrm{L}_{\mathrm{n}}=513 \mu \mathrm{m} \\
& \mathrm{W}_{\mathrm{n}}=309 \mu \mathrm{m} \\
& \text { Area }=513 \times 309 \mu \mathrm{m} 2=158730 \mu \mathrm{m}^{2}=0.159 \mathrm{~mm}^{2} .
\end{aligned}
$$

Area reduction:

$$
\% \text { Area reduction: }((0.21-0.159) \times 100) \div 0.21=24.28 \%
$$

This reduction in the chip area has been possible primarily due the use of MBE, which reduces the partial products by half. The logic for the removal of the last 
neg signal does not contribute to the overall area reduction because the overhead of 2's complement logic and 5-1 selector dominates over the gain of reducing one compressor. 


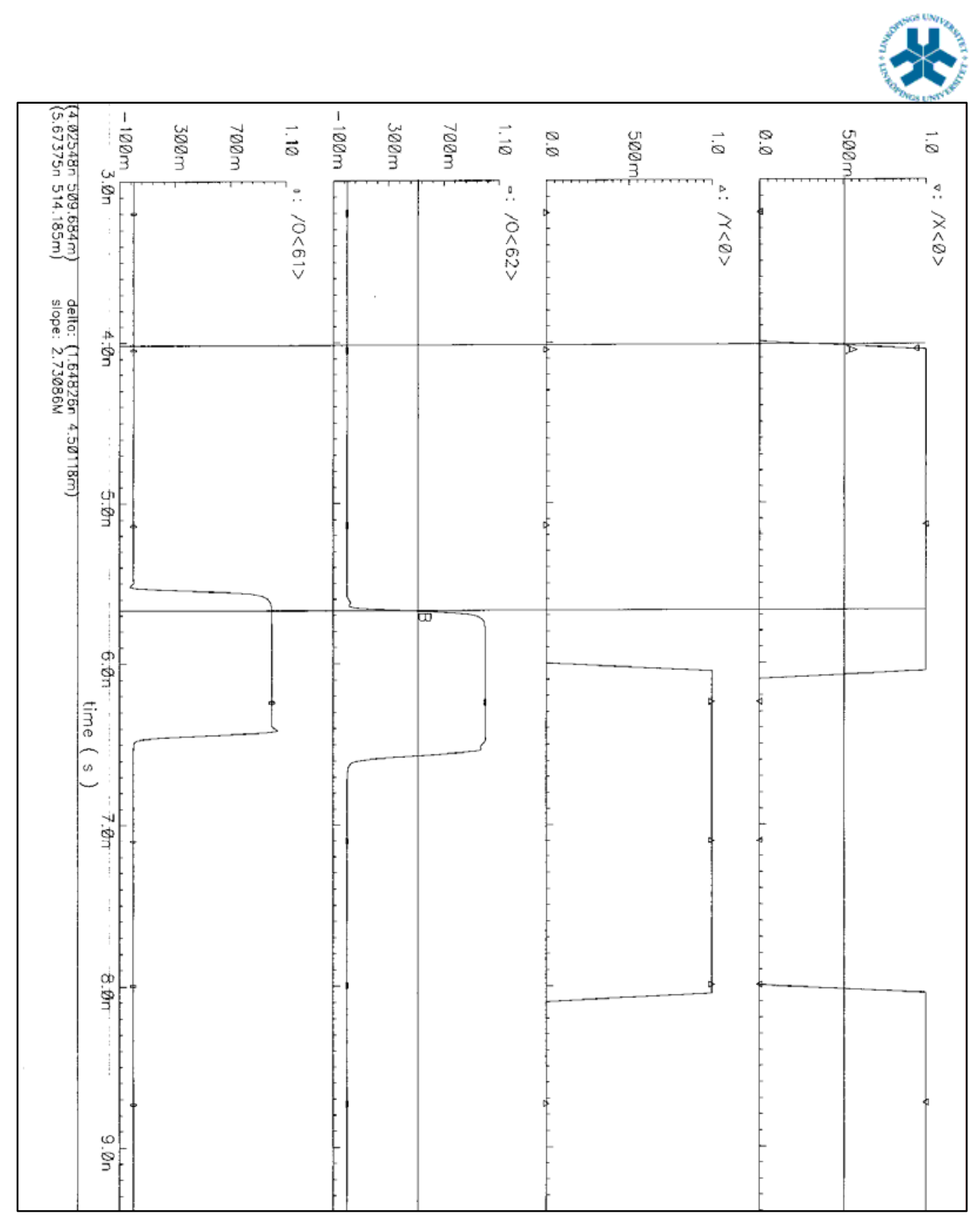

Figure 55: Simulation results @1.0V 


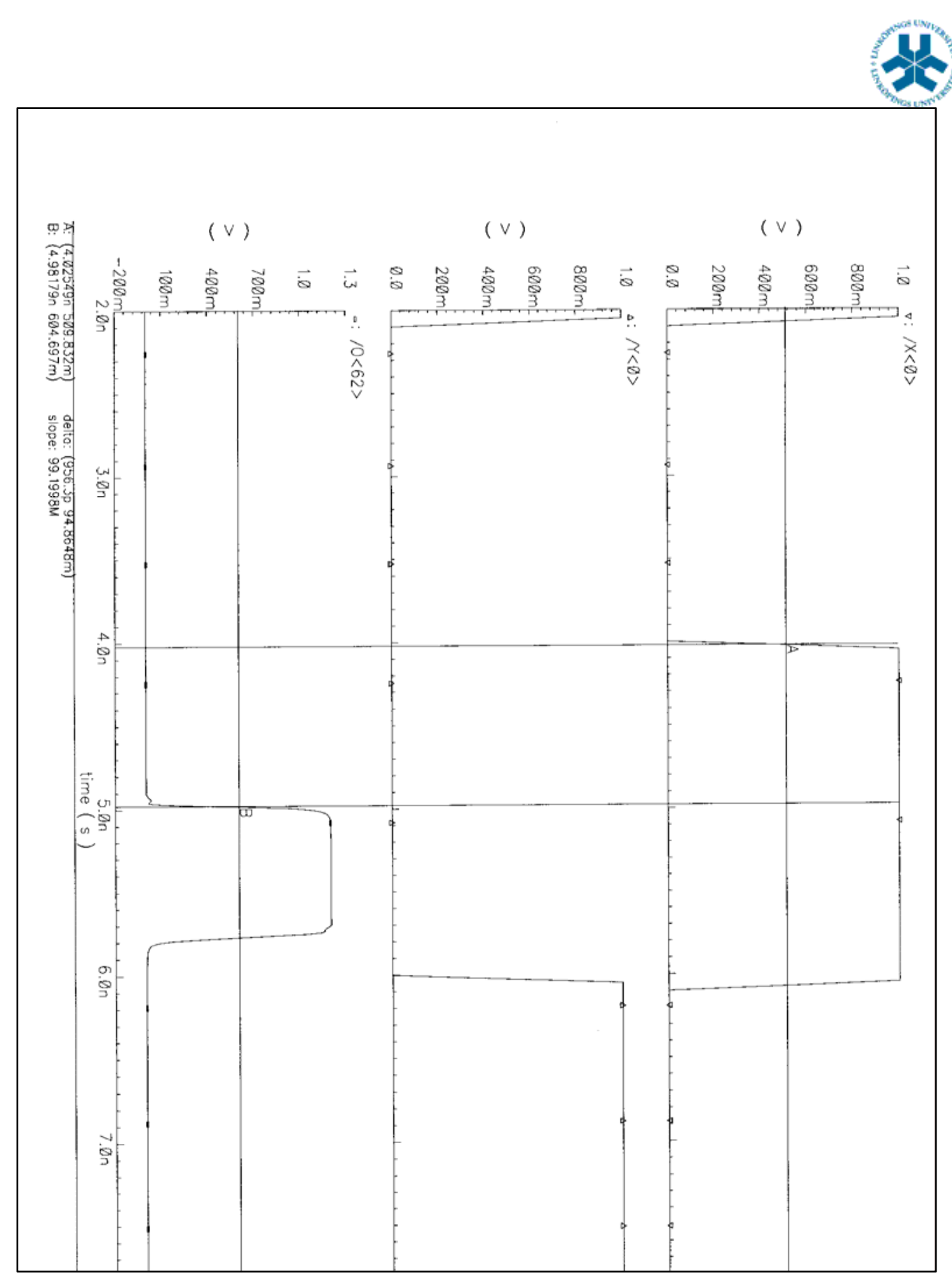

Figure 56: Simulation results @ 1.2V 


\subsection{Future work direction}

The present work on the new multiplier architecture can be extended in various directions. Some suggestions are given below.

1. In order to completely analyze the performance, the layout can be extended to chip level where the delays due to wiring, interconnects and PAD are included.

2. The multiplier can be designed in some other CMOS logic like Domino logic or Complementary Pass transistor Logic (CPL) etc. some logic families are faster than static CMOS logic, so it is important to analyze the performance for those families.

3. The adder tree structure can be changed by the use of other trees like delay-balanced, binary tree or overturned-staircase tree.

\subsection{Conclusions}

In this thesis report energy-efficient 32-bit multiplier architecture has been presented. The architecture is based on a modified Booth-encoding scheme, which reduces the number of partial-products by half compared to a conventional implementation. Simulation results show that for equal delay the power-efficiency of the proposed architecture is improved by $47 \%$ and the area is reduced by $24 \%$ compared to a conventional implementation. 
* 


\section{PART VII}

\section{REFERENCES}


* 


\section{References}

\section{Books}

[1]

[2]

[3]

[4]

Jan M. Rabaey, Anantha Chansrakasan, Borivoje Nikolic, "Digital Integrated Circuits", $2^{\text {nd }}$ Edition, Prentice Hall Electronics and VLSI series.

2] Michael J.Flynn, Stuart F.Oberman, "Advanced Computer Arithmetic Design" A Wiley-Interscience publication.

Behrooz Parhami, "Computer Arithmetic, algorithms and hardware designs" Oxford University press 2000.

Lars Wanhalmer ,"Digital Signal Processing" Chapter 11 'Processing Elements'.

\section{Research Papers}

[5] Jung-Yup Kang, Jean-Luc Gaudiot, "A fast and well-structured multiplier", proceedings of the EUROMICRO systems on Digital System Design (DSD’04) IEEE.

[6] Anantha P. Chandrakasan and Rebert W. Brodersen,"Minimizing Power consumption in digital CMOS circuits" proceedings of the IEEE Vol. 83 No. 41995.

[7] D. Bakalis, E. Kalligeros, D. Nikolos " Low power BIST for Wallace tree based fast multipliers" First International Symosium on Quality of Electronic design, 2000.

[8] Moises E. Robinson, Earl Swartzlander "A reduction scheme to optimize the Wallace multiplier" IEEE International Conference on Computer Design (ICCD) 1998.

[9] Kwen-Siong Chong, Bah-Hwee, Joseph Chang "A micropower low voltage multiplier with reduced spurious switching" IEEE Transactions on VLSI Systems, Vol. 13, No. 2, 2005.

[10] King Fai Pang "Architectures for pipelined Wallace tree multiplier-accumulators" IEEE International Conference on VLSI in computers and processors 1990 (ICCD'90).

[11] M.J.Liao, C.F.Su, Chang and Allen Wu "A carry select adder optimization technique for high-performance Booth-encoded Wallace tree multipliers" IEEE International Symposium on Circuits and Systems 2002.

[12] M. D. Ercegovac and T. Lang. "Digital Arithmetic" Morgan Kaufmann Publishers, Los Altos, CA 94022, USA, 2003. 


\section{Web links}

[13]

[14]

[15]

$[16]$

$[17]$

$[18]$

[19]

[20]

[21]
(June 2006) Wayne Wolf www.princeton.edu/ wolf/modern-vlsi/overheads/CHAP6-2.

(July 2006) www.aoki.ecei.tohoku.ac.jp/arith/mg/algorithm.html\#fsa_csa

www.zeuthen.desy.de/ape/html/APEmille/documentation/hardware/Tmille/talu_ch9.pdf

(July, 2006) http://en.wikipedia.org/wiki/wallace tree

(Aug 2006) www.cs.wisc.edu/arch/www/ISCA-2000-panel/T.N.vijaykumar/sld003.htm

http://bwrc.eecs.berkeley.edu/publoications/theses/low.power.cmos.library.MS/power2.html

(Aug 2006) http://en.wikipedia.org/wiki/clock gating

(Aug 2006) http://research.microsoft.com/ lorch/papers/mobisys2003/node4.html

(Aug 2006) http://www.es.isy.liu.se/courses/TSTE85/local/S-041215.pdf 
På svenska

Detta dokument hålls tillgängligt på Internet - eller dess framtida ersättare under en längre tid från publiceringsdatum under förutsättning att inga extraordinära omständigheter uppstår.

Tillgång till dokumentet innebär tillstånd för var och en att läsa, ladda ner, skriva ut enstaka kopior för enskilt bruk och att använda det oförändrat för ickekommersiell forskning och för undervisning. Överföring av upphovsrätten vid en senare tidpunkt kan inte upphäva detta tillstånd. All annan användning av dokumentet kräver upphovsmannens medgivande. För att garantera äktheten, säkerheten och tillgängligheten finns det lösningar av teknisk och administrativ art.

Upphovsmannens ideella rätt innefattar rätt att bli nämnd som upphovsman i den omfattning som god sed kräver vid användning av dokumentet på ovan beskrivna sätt samt skydd mot att dokumentet ändras eller presenteras i sådan form eller i sådant sammanhang som är kränkande för upphovsmannens litterära eller konstnärliga anseende eller egenart.

För ytterligare information om Linköping University Electronic Press se förlagets hemsida http://www.ep.liu.se/

In English

The publishers will keep this document online on the Internet - or its possible replacement - for a considerable time from the date of publication barring exceptional circumstances.

The online availability of the document implies a permanent permission for anyone to read, to download, to print out single copies for your own use and to use it unchanged for any non-commercial research and educational purpose. Subsequent transfers of copyright cannot revoke this permission. All other uses of the document are conditional on the consent of the copyright owner. The publisher has taken technical and administrative measures to assure authenticity, security and accessibility.

According to intellectual property law the author has the right to be mentioned when his/her work is accessed as described above and to be protected against infringement.

For additional information about the Linköping University Electronic Press and its procedures for publication and for assurance of document integrity, please refer to its WWW home page: http://www.ep.liu.se/

(C) [Nasir Mehmood] 
* 